(C) 2017. This manuscript version is made available under the CC-BY-NC-ND 4.0 license http://creativecommons.org/ licenses/by-nc-nd/4.0/

\title{
Neural Network Adaptive Control Design for Robot Manipulators under Velocity Constraints
}

\author{
Hamed N. Rahimi*, Ian Howard, and Lei Cui \\ Faculty of Science and Engineering, School of Civil and Mechanical Engineering, Curtin University, Bentley, WA 6102, \\ Australia
}

\begin{abstract}
This paper studies the neural adaptive control design for robotic systems with uncertain dynamics under the existence of velocity constraints and input saturation. The control objective is achieved by choosing a control Lyapunov function using joint error variables that are restricted to linear growth and furthermore by introducing a secant type barrier Lyapunov function for constraining the joint rate variables. The former is exploited to bind the forward propagation of the position errors, and the latter is utilized to impose hard bounds on the velocity. Effective input saturation is expressed, and neural networks are employed to tackle the uncertainty problem in the system dynamics. Feasibility conditions are formulated, and the optimal design parameters are obtained by solving the constrained optimization problem. We prove that under the proposed method, semi-global uniform ultimate boundedness of the closed-loop system can be guaranteed. Tracking errors meanwhile converge to small neighborhoods of the origin, and violations of predefined velocity constraints are avoided. Finally, numerical simulations are performed to verify the effectiveness of the theoretical developments.
\end{abstract}

Keywords: input saturation, radial basis function neural network, secant type barrier Lyapunov function, uncertain robots, velocity constraint

\section{Introduction}

Adaptive constrained control (ACC) has made great progress in recent robotic studies, driven by practical needs coupled with the ability to overcome theoretical challenges. Indeed, this method recently has gained significant importance due to its ability to improve safety and to reduce dependency on accurate knowledge of the system dynamics. Based on the Lyapunov stability theory, ACC has been extensively developed for robotic systems employing methods like adaptive position/force control, coordinated control, adaptive vision and force tracking control, admittance control, and impedance control.

Barrier Lyapunov Functions (BLF) have been developed as a result of studies concerning how the control Lyapunov function (CLF) can be shaped to bound the states or suppress the propagation of the system error so as to achieve ACC. In the 2004 and 2005 seminal works [1,2], Ngo et al. pointed out that the barrier function's characteristics can be employed to shape the structure of the CLF. Such a function grows to infinity whenever its arguments approach some limits. Inspired by this idea, Tee et al. [3, 4] developed BLF for control of the system with the output constraints. This method relies on bounding of the Lyapunov function in the closed loop, to ensure that the constraints are not transgressed. Starting from then, many papers used BLF to control the dynamic systems with output [5], and full state [6-9] constraints. Accordingly, BLF have been extensively studied for ACC of robots [9-11] and various practical systems such as flexible structure systems like [12] where a hybrid PDEODE system was considered and methods like boundary control were utilized to solve the problem. Other examples are autonomous surface vessels, wind turbines and teleoperation systems [13-15].

Nevertheless, due to its shaping to comprise the CLF, BLF-based control may increase the magnitude of the control signal remarkably as the barrier limits are approached. Neglecting this effect can cause dangerous conditions in robotic applications, specifically when the robot is collaborating closely with the human. In addition, failure to bound the input torque may result in undesirable inaccuracy, system instability or performance degradation [16, 17]. Thus, physical input saturation could be encountered during attempts to provide system reliability, and safety in operation. A review of the literature shows that several

${ }^{*}$ Correspondence to: Faculty of Science and Engineering, School of Civil and Mechanical Engineering, Curtin University, Bentley, WA 6102, Australia.

E-mail addresses: hamed.rahiminohooji@postgrad.curtin.edu.au (H.N. Rahimi), i.howard@curtin.edu.au (I. Howard), lei.cui@curtin.edu.au (L. Cui). 
methods like adaptive control, model predictive control, low-gain control, neural network control and antiwindup compensator are dealt with by the system with the input saturation. However, to our knowledge, only limited research works have considered the problem of constraining the input control in the design of the BLF-based control systems [18].

In reality, many industrial robotic tasks involve dynamic forces such as Coriolis and centrifugal forces that vary as a function of the square of the speed. Thus, if the robot attempts to move too quickly, it will cause a large dynamic force due to a high joint rate or velocity. Accordingly, a constrained stable control strategy is required to keep the speed of robot motion low, so as to avoid failure of the closed-loop system. In fact, in several practical industrial applications, e.g., robotic applications, position rates must be bounded below some specific bounds to avoid saturation, while position constraints may not be necessary. On the other hand, in many industrial robotic systems, the controller's accuracy may quickly degrade as the speed of motion increases. Hence, bounding the velocity can improve accuracy in robot tasks. Recent categories of practical robotic systems that need to operate with constrained velocity include robotic applications that have close interactions with humans, where safety becomes a critical issue. Examples are social robots, robotic surgery, and the safe robotic rehabilitation. The main focus of this paper is concerned with how to limit the robot joint velocities by designing an effective control law, and further how to compensate for the robot torques' saturation characteristic.

Motivated by the above-mentioned considerations, this paper presents BLF-based velocity constrained control of robotic systems with input saturation, and unknown dynamics and unknown interaction forces. The primary objective of the paper is to study stable ACC design for uncertain robotic systems subject to velocity constraints. An adaptive neural control design approach is presented for uncertain robotic systems considering velocity constraints. In the control design, radial basis function (RBF) neural networks $(\mathrm{NN})$ are utilized to handle uncertainties, and secant type barrier Lyapunov functions are introduced to develop a novel constrained adaptive control scheme. Feasibility conditions involving the initial states and control parameters selection are formulated and based on Lyapunov theory, the stability is proven, and the boundedness of all closed-loop systems is guaranteed.

Compared with the available studies, the main contributions of this paper can be summarized as follows.

1) To the best of our knowledge, this is the first time in the literature that BLF is utilized for velocity constrained tracking control of the robotic system without considering extra constraints on joint positions.

2) A novel BLF, named " $s B L F$ ", is introduced by reshaping the CLF, which guarantees that the preferred variables remain in their respective constraint sets.

3) Proper input saturation is adopted, and offline feasibility checking is utilized using the constrained optimization algorithm. Then, by introducing Lemma 2, via neural ACC, uniformly ultimate boundedness of the closed-loop system is proven.

4) In addition, compared with the existing literature, the presented method removed the extra steps on mapping [19], error transformation [20], or transforming the constrained system into the unconstrained one [21], by directly exploiting the constraints on the control design.

Throughout this paper, we use $\mathbf{R}$ and $\mathbf{R}^{+}$to denote the sets of real numbers and non-negative real numbers, respectively. $(\tilde{\bullet})=(\bullet)-(\hat{\bullet})$, with $(\hat{\bullet})$ represents the estimated value of $(\bullet)$, vertical bars $\|\bullet\|$ represent the Frobenius norm for matrices or the Euclidean norm for vectors, and $\lambda_{\min }(\bullet)$ and $\lambda_{\max }(\bullet)$ denote the smallest and largest eigenvalues of a square matrix $(\bullet)$, respectively. We use $\operatorname{sign}(\bullet)$ to denote the standard decentralized unit sign function.

\section{Preliminaries and problem formulation}

\subsection{Useful technical lemmas and definitions}

Lemma 1: In this paper, we present a novel Lyapunov function with a barrier function characteristics as,

$$
\boldsymbol{V}=\sec \left(\frac{\pi \chi^{2}}{2 \boldsymbol{k}_{\chi}^{2}}\right)-1,|\chi(0)|<\boldsymbol{k}_{\chi},
$$


where $\boldsymbol{k}_{\chi}$ is the desired bound, and $\chi$ is the variable that needed to be constrained such that $|\chi(\boldsymbol{t})|<\boldsymbol{k}_{\chi}$. The BLF presented at (1) is positive definite and $\boldsymbol{C}^{1}$ continuous in the set $|\chi(\boldsymbol{t})|<\boldsymbol{k}_{\chi}$ with a growth condition governed by,

$$
|\chi| \rightarrow \boldsymbol{k}_{\chi} \Rightarrow \boldsymbol{V} \rightarrow \infty
$$

In this work, by incorporating the proposed secant-type BLF in (1), which we name " $B L F$ ", into the Lyapunov function design procedure, we will guarantee the boundedness on the velocity variable, and hence satisfy the robot velocity constraint requirement.

Lemma 2:

a. The following inequality holds for all $\boldsymbol{x} \in \mathbf{R}$,

$$
\sqrt{1+x^{2}}-1 \leq \frac{x^{2}}{\sqrt{1+x^{2}}}
$$

b. The following inequality holds for any $\boldsymbol{x}$ in the interval $|\boldsymbol{x}|<1$,

$$
\sec \left(\frac{\pi}{2} x^{2}\right)-1 \leq \pi x^{2} \tan \left(\frac{\pi}{2} x^{2}\right) \sec \left(\frac{\pi}{2} x^{2}\right)
$$

Proof:

a. Let $\Xi(x)=x^{2} / \sqrt{1+x^{2}}-\sqrt{1+x^{2}}+1$. The derivation of $\Xi(x)$ with respect to $x$ is given by $d \Xi(x) / d x=x /\left(x^{2}+1\right)^{3 / 2}$. It is obvious that $(d \Xi(x) / d x)<0$, for $x<0, \quad(d \Xi(x) / d x)=0$, for $x=0$, and $(d \Xi(x) / d x)>0$, for $x>0$. Accordingly, considering $\Xi(0)=0$ it can be shown that $\Xi(x) \geq 0$ and furthermore it proves that $x^{2} / \sqrt{1+x^{2}} \geq \sqrt{1+x^{2}}-1$.

b. Let $\Xi_{1}(x)=\pi x^{2} \tan \left(\pi x^{2} / 2\right) \sec \left(\pi x^{2} / 2\right)-\sec \left(\pi x^{2} / 2\right)+1$, and $\Xi_{2}(x)=\Xi_{1}(x) / \sec ^{2}\left(\pi x^{2} / 2\right)$; then we have $\Xi_{2}(x)=\pi x^{2} \sin \left(\pi x^{2} / 2\right)-\cos \left(\pi x^{2} / 2\right)+\cos ^{2}\left(\pi x^{2} / 2\right)$. The derivation of $\Xi_{2}(x)$ with respect to $x$ is given by $d \Xi_{2}(x) / d x=\pi x\left(3 \sin \left(\pi x^{2} / 2\right)-\sin \left(\pi x^{2}\right)+\pi x^{2} \cos \left(\pi x^{2} / 2\right)\right)$. Therefore

$d \Xi_{2}(x) / d x=\pi x\left(\sin \left(\pi x^{2} / 2\right)\left(3-2 \cos \left(\pi x^{2} / 2\right)\right)+\pi x^{2} \cos \left(\pi x^{2} / 2\right)\right)$.

Since for any $x \in(-1,1)$, we have $0 \leq \sin \left(\pi x^{2} / 2\right)<1$, and $0 \leq \cos \left(\pi x^{2} / 2\right)<1$, then it is obvious that $\left(d \Xi_{2}(x) / d x\right)<0$, for $\boldsymbol{x}<0, \quad\left(d \Xi_{2}(x) / d x\right)=0$, for $\boldsymbol{x}=0$, and $\left(\boldsymbol{d} \Xi_{2}(\boldsymbol{x}) / \boldsymbol{d} \boldsymbol{x}\right)>0$, for $\boldsymbol{x}>0$. Accordingly, considering $\Xi_{2}(0)=0$ it can be obtained that $\Xi_{2}(\boldsymbol{x}) \geq 0$ and furthermore it proves $\Xi_{1}(\boldsymbol{x}) \geq 0$, and consequently the inequality (3) is proved.

Lemma 3 [22]: For any arbitrary $\boldsymbol{\mu} \in \mathbf{R}$ and $\boldsymbol{v}>0$, the following inequality can be established,

$$
0 \leq|\boldsymbol{\mu}|-\boldsymbol{\mu} \tanh \left(\frac{\boldsymbol{\mu}}{\boldsymbol{v}}\right) \leq 0.2785 \boldsymbol{v}
$$

This Lemma is employed to deal with some uncertainties that arise in the control design procedure.

Lemma 4[18]: Consider the smooth continuous function $\Theta(\boldsymbol{t})$ for $\boldsymbol{t} \in\left[\boldsymbol{t}_{0}, \boldsymbol{t}_{1}\right]$ that is bounded with bounded $\boldsymbol{t}_{0}$ and $\boldsymbol{t}_{1}$, providing $\varsigma_{1} \leq\|\Theta\| \leq \varsigma_{2}$ where $\varsigma_{1}$ and $\varsigma_{2}$ are the positive constants. Then, the boundness of $\dot{\Theta}(\boldsymbol{t})$ is guaranteed.

Definition 1 [23]: The solution of a system $\boldsymbol{X}(\boldsymbol{t})$ is Semiglobally Uniformly Ultimately Bounded (SGUUB) if, for any compact set $\Omega_{0}$ and all $\boldsymbol{X}\left(\boldsymbol{t}_{0}\right) \in \Omega_{0}$, there exists a $\lambda>0$ and a number $\boldsymbol{T}\left(\boldsymbol{\lambda}, \boldsymbol{X}\left(\boldsymbol{t}_{0}\right)\right)$ such that $\|\boldsymbol{X}(\boldsymbol{t})\| \leq \boldsymbol{\lambda}$ for all $\boldsymbol{t} \geq \boldsymbol{t}_{0}+\boldsymbol{T}$. 
Due to its learning abilities, and capabilities in function approximation, in this study, RBF NN [24] was employed to approximate any continuous function $\boldsymbol{f}(\boldsymbol{Z}): \mathbf{R}^{\boldsymbol{m}} \rightarrow \mathbf{R}$ as,

$$
f_{r b f}(Z)=\omega^{T} h(Z)
$$

where $\boldsymbol{Z} \in \Omega_{z} \subset \mathbf{R}^{\boldsymbol{m}}$ is the NN input vector with $\boldsymbol{m}$ being the NN input dimension, $\boldsymbol{\omega} \in \mathbf{R}^{\boldsymbol{r}}$ is the weight vector, $\boldsymbol{r}>1$ is the NN node number, $\boldsymbol{h}(\boldsymbol{Z})=\left[\boldsymbol{h}_{1}(\boldsymbol{Z}), \boldsymbol{h}_{2}(\boldsymbol{Z}), \ldots, \boldsymbol{h}_{\boldsymbol{l}}(\boldsymbol{Z})\right]^{\boldsymbol{T}}$ is a basis function vector with $\boldsymbol{h}_{\boldsymbol{i}}(\boldsymbol{Z})$ for $\boldsymbol{i}=1, \ldots, \boldsymbol{l}$, being the Gaussian functions that can be expressed as $\boldsymbol{h}_{\boldsymbol{i}}(\boldsymbol{Z})=\exp \left(-\left(\boldsymbol{Z}-\boldsymbol{\vartheta}_{\boldsymbol{i}}\right)^{\boldsymbol{T}}\left(\boldsymbol{Z}-\boldsymbol{\vartheta}_{\boldsymbol{i}}\right) / \boldsymbol{\psi}^{2}\right)$, with $\boldsymbol{\psi}$ being the width of the Gaussian functions, and $\vartheta_{i}=\left[\vartheta_{i 1}, \vartheta_{i 2}, \ldots, \vartheta_{i m}\right]^{T}$ being the center of the $\boldsymbol{i}^{\text {th }}$ input element of the NN. Also, using [25] it can be shown that for the Gaussian RBF NN (4), there exists a constant $\boldsymbol{C}_{1}>0$ such that $\|\boldsymbol{h}(\boldsymbol{Z})\| \leq \boldsymbol{C}_{1}$. In [26], it has been indicated that by choosing the sufficiently large number of nodes, the RBF NN (4) can approximate any continuous function $\boldsymbol{f}(\boldsymbol{Z})$ over the compact set $\Omega_{z} \subset \mathbf{R}^{m}$ to an arbitrary accuracy $\varepsilon_{M}$ as $\boldsymbol{f}(\boldsymbol{Z})=\boldsymbol{\omega}^{* T} \boldsymbol{h}(\boldsymbol{Z})+\boldsymbol{\varepsilon}(\boldsymbol{Z}), \forall \boldsymbol{Z} \in \Omega_{z} \subset \mathbf{R}^{\boldsymbol{m}}$, where $\boldsymbol{\omega}^{*}$ is the ideal constant weight vector, and $\boldsymbol{\varepsilon}(\boldsymbol{Z})$ is the unknown approximation error.

Assumption 1 [27]: For a given continuous function $\boldsymbol{h}(\boldsymbol{Z})$ and RBF NN approximator (4), there exist optimal constant weights $\omega^{*}$ such that the reconstruction error $\boldsymbol{\varepsilon}(\boldsymbol{Z})$ is upper bounded in the sense that $\|\boldsymbol{\varepsilon}(\boldsymbol{Z})\| \leq \boldsymbol{\varepsilon}_{\boldsymbol{M}}, \forall \boldsymbol{Z}, \in \Omega_{z} \subset \mathbf{R}^{\boldsymbol{m}}$ with $\boldsymbol{\varepsilon}_{M} \in \mathbf{R}^{+}$being an unknown constant.

The following Lemma shows that there exists an upper bound on the basis function vector in (4). This Lemma will be used to show the boundedness of the designed control of the closed-loop system.

For simplifying notation, from this point onwards, the state and time dependence of the system is omitted, whenever possible without creating confusion.

\subsection{System description and problem formulation}

The dynamical equation of an $\boldsymbol{n}$ dimensional serial fully-actuated robotic manipulator can be described as,

$$
\boldsymbol{M}(\boldsymbol{q}) \ddot{\boldsymbol{q}}+\boldsymbol{C}(\boldsymbol{q}, \dot{\boldsymbol{q}}) \dot{\boldsymbol{q}}+\boldsymbol{G}(\boldsymbol{q})=\mathrm{T}(\boldsymbol{\tau})+\boldsymbol{\Gamma}
$$

where $\boldsymbol{q}, \dot{\boldsymbol{q}}, \ddot{\boldsymbol{q}} \in \mathbf{R}^{\boldsymbol{n}}$ are the position, velocity, and acceleration vectors, respectively, $\boldsymbol{M}(\boldsymbol{q}) \in \mathbf{R}^{\boldsymbol{n} \times \boldsymbol{n}}$ denotes the inertia matrix, $\boldsymbol{C}(\boldsymbol{q}, \dot{\boldsymbol{q}}) \in \mathbf{R}^{\boldsymbol{n} \times \boldsymbol{n}}$ represents the centrifugal and Coriolis forces matrix, $\boldsymbol{G}(\boldsymbol{q}) \in \mathbf{R}^{\boldsymbol{n}}$ is the gravitational force/torque vector; $\boldsymbol{\tau} \in \mathbf{R}^{\boldsymbol{n}}$ is the desired continuous control input vector, $T(\tau) \in \mathbf{R}^{n}$ is a vector of saturation limits for the joint torque $\tau$, and $\Gamma(t) \in \mathbf{R}^{n}$ is the force exerted by the human and environment, uniformly bounded by unknown constant $\boldsymbol{\Gamma}_{\boldsymbol{M}} \in \mathbf{R}^{+}$, such that $|\boldsymbol{\Gamma}(\boldsymbol{t})| \leq \boldsymbol{\Gamma}_{M}, \forall \boldsymbol{t} \in[0, \infty)$.

Property 1: The inertia matrix $\boldsymbol{M}(\boldsymbol{q})$ is symmetric and positive definite. Further, the matrix $\dot{\boldsymbol{M}}(\boldsymbol{q})-2 \boldsymbol{C}(\boldsymbol{q}, \dot{\boldsymbol{q}})$ is skewsymmetric.

The input saturation constraint signal vector $\mathrm{T}(\tau)$ is expressed as,

$$
\mathrm{T}(\tau)=\mathrm{T}_{\max } \tanh \left(\frac{\tau}{\mathrm{T}_{\max }}\right)
$$

where $\mathrm{T}_{\max } \in \mathbf{R}^{\boldsymbol{n}}$ is the known upper bound vector on the actuator.

Remark 1: Input saturation functions designed in [28-30] using the sign function as $\mathrm{T}(\tau)=\operatorname{sign}(\tau) \mathrm{T}_{\max }$ if $|\tau| \geq \mathrm{T}_{\max }$; 
otherwise $\mathrm{T}(\tau)=\tau$, have sharp corners as $|\tau|=\mathrm{T}_{\max }$. Nevertheless, since the backstepping technique requires all functions to be differentiable, this relationship between $\mathrm{T}_{\max }$ and $\tau$ may possibly cause a problem for the backstepping technique to be directly applied. However, the presented function (6) employed the hyperbolic tangent function to approximate the input saturation which provided a smooth function avoiding the problem.

The robot dynamic equation presented by (5), can be re-expressed as,

$$
\begin{aligned}
& \dot{\boldsymbol{x}}_{1}=\boldsymbol{x}_{2} \\
& \dot{\boldsymbol{x}}_{2}=\boldsymbol{M}^{-1}\left(\mathrm{~T}(\tau)+\boldsymbol{\Gamma}-\boldsymbol{C} \boldsymbol{x}_{2}-\boldsymbol{G}\right)=\boldsymbol{a},
\end{aligned}
$$

where $\boldsymbol{x}_{1}=\boldsymbol{q}=\left[\boldsymbol{q}_{1}, \boldsymbol{q}_{2}, \ldots, \boldsymbol{q}_{n}\right]^{T}$, and $\boldsymbol{x}_{2}=\dot{\boldsymbol{q}}=\left[\dot{\boldsymbol{q}}_{1}, \dot{\boldsymbol{q}}_{2}, \ldots, \dot{\boldsymbol{q}}_{n}\right]^{T}$.

The main objective of this paper is to design an adaptive controller for the robot dynamic system given by (5) under the existence of velocity constraints where $\dot{\boldsymbol{q}}(\boldsymbol{t})$ remains in the constrained region $\Omega_{v}=\left\{\dot{\boldsymbol{q}}_{\boldsymbol{i}} \in \mathbf{R}, \boldsymbol{i}=1, \ldots, \boldsymbol{n} \| \dot{\boldsymbol{q}}_{i}(\boldsymbol{t}) \mid<\boldsymbol{k}\right.$ i $\left.(\boldsymbol{t}), \boldsymbol{t} \geq 0\right\}$, i.e., $\dot{\boldsymbol{q}}(\boldsymbol{t}) \in \Omega_{\boldsymbol{v}}, \boldsymbol{t}>0$, provided $\dot{\boldsymbol{q}}(0) \in \Omega_{\imath}$, such that all the signals in the closed-loop system remain bounded and robot joint positions follow the given desired trajectories $\boldsymbol{x}_{d}(\boldsymbol{t})=\left[\boldsymbol{x}_{d 1}, \boldsymbol{x}_{d 2}, \ldots, \boldsymbol{x}_{d n}\right]^{T}$ as closely as possible, i.e., $\lim _{\boldsymbol{t} \rightarrow \infty}\left|\boldsymbol{q}_{i}(\boldsymbol{t})-\boldsymbol{x}_{d i}(\boldsymbol{t})\right|=\boldsymbol{t}_{i}$ with $\boldsymbol{t}_{\boldsymbol{i}}$ being a small positive constant.

Assumption 2 [31]: The desired trajectory $\boldsymbol{x}_{\boldsymbol{d}}(\boldsymbol{t})$ and its first time derivative $\dot{\boldsymbol{x}}_{\boldsymbol{d}}(\boldsymbol{t})$ are continuous and bounded. Also, there exist positive constants $\overline{\boldsymbol{x}}_{d i}$ and $\overline{\boldsymbol{x}}_{d 1 i}, \boldsymbol{i}=1, \ldots, \boldsymbol{n}$, such that $\left|\boldsymbol{x}_{d i}(\boldsymbol{t})\right| \leq \overline{\boldsymbol{x}}_{d i}$ and $\left|\dot{\boldsymbol{x}}_{d i}(\boldsymbol{t})\right| \leq \overline{\boldsymbol{x}}_{d 1 i}, \forall \boldsymbol{t}>0$.

\section{Controller design and stability analysis}

The objective of this section is to design the controller that bounds the velocity of the robotic system with unknown dynamics. First, the Lyapunov function is chosen to impose a bound on the propagation of the error in the position stage. To do this, choose a Lyapunov function candidate as,

$$
V_{1}=\sum_{i=1}^{n} \boldsymbol{k}_{1 i}\left(\sqrt{1+\boldsymbol{e}_{i}^{2}}-1\right)
$$

where, $\boldsymbol{k}_{1 i}, \boldsymbol{i}=1, \ldots, \boldsymbol{n}$ are positive design parameters, and $\boldsymbol{e}_{\boldsymbol{i}}=\boldsymbol{x}_{1 i}-\boldsymbol{x}_{d i}$, for $\boldsymbol{i}=1, \ldots, \boldsymbol{n}$, denote the position error variables. Note that using the Lyapunov function (8), and choosing a small amount of $\boldsymbol{k}_{1 i}$, the growth of the Lyapunov function in the position stage can be restricted to a linear growth or less.

Let the variable transformation $\boldsymbol{z} \in \mathbf{R}^{\boldsymbol{n}}$ satisfy $\boldsymbol{z}=\boldsymbol{x}_{2}-\boldsymbol{\alpha}$, where $\boldsymbol{\alpha} \in \mathbf{R}^{\boldsymbol{n}}$ is a vector of the virtual control signal being designed. It can be verified simply from (8) that,

$$
\dot{V}_{1}=\sum_{i=1}^{n} \frac{\boldsymbol{k}_{1 i} \boldsymbol{e}_{i}}{\sqrt{1+\boldsymbol{e}_{i}^{2}}}\left(z_{i}+\alpha_{i}-\dot{\boldsymbol{x}}_{d_{i}}\right)
$$

Choose the virtual control $\alpha$ as,

$$
\alpha_{i}=-c_{1 i} \tanh \left(\boldsymbol{e}_{i}\right)+\dot{\boldsymbol{x}}_{d_{i}}
$$

where $\boldsymbol{c}_{1 i}>0$ is a positive constant. From (10), and Assumption 2, it is easy to obtain $\left|\boldsymbol{\alpha}_{i}\right| \leq \overline{\boldsymbol{\alpha}}_{i}$ with $\overline{\boldsymbol{\alpha}}_{i}=\boldsymbol{c}_{1 i}+\overline{\boldsymbol{x}}_{\boldsymbol{d} 1 i}$. Boundedness of $\alpha_{i}$ in this stage will help to satisfy the control goal of bounding the velocity $\boldsymbol{x}_{2 i}$ in the rest of the control design. Substituting (10) into (9) results in,

$$
\dot{V}_{1}=-\sum_{i=1}^{n} c_{1 i} \boldsymbol{k}_{1 i} \frac{\boldsymbol{e}_{i} \tanh \left(\boldsymbol{e}_{i}\right)}{\sqrt{1+\boldsymbol{e}_{i}^{2}}}+\sum_{i=1}^{n} \frac{\boldsymbol{k}_{1 i} \boldsymbol{e}_{i}}{\sqrt{1+\boldsymbol{e}_{i}^{2}}} z_{i}
$$


Remark 2: As the term $c_{1} \boldsymbol{k}_{1} \boldsymbol{e} \tanh (\boldsymbol{e}) / \sqrt{1+\boldsymbol{e}^{2}}$ is positive-definite in $\boldsymbol{e}$, it is obvious from (11) that $\dot{\boldsymbol{V}}_{1}$ becomes negativedefinite once $z=0$. However, for the case that the variable transformation $z$ is not driven as zero, the error would propagate through to the system via the second term on the right-hand side of (11), if the position error, $\boldsymbol{e}$, is sufficiently large. In this study, the growth of the Lyapunov function as a result of the position error is limited by using the Lyapunov function with linear growth in (8). By that means, the forward propagation of the position error through to the rest of the control design procedure is prevented.

By ensuring the boundedness of the forward propagation of the position error, we are now ready to impose a hard-bound to the variable transformation $z$. To do this, we adopt the following Lyapunov function based on the presented sBLF,

$$
V_{2}=V_{1}+\frac{1}{2} z^{T} \boldsymbol{M z}+\sum_{i=1}^{n} \sec \left(\frac{\pi z_{i}{ }^{T} z_{i}}{2 \boldsymbol{k}_{c i}{ }^{2}}\right)-1
$$

Note that when using the SBLF term in (12), the Lyapunov function $\boldsymbol{V}_{2}$ will approach infinity as $\left|\boldsymbol{z}_{\boldsymbol{i}}\right| \rightarrow \boldsymbol{k}_{c i}$. Thus, such a choice of $\boldsymbol{V}_{2}$ yields $\left|\boldsymbol{z}_{i}\right|<\boldsymbol{k}_{\boldsymbol{c}}, \forall \boldsymbol{t} \geq 0$. Further, since $\boldsymbol{z}_{i}$, and $\boldsymbol{\alpha}_{i}$ are bounded, the joint velocity variable $\dot{\boldsymbol{q}}=\boldsymbol{x}_{2}$ is consequently bounded as $\boldsymbol{x}_{2}=\boldsymbol{z}+\boldsymbol{\alpha}$ with over-bound on $\left|\dot{\boldsymbol{q}}_{i}\right| \leq\left|\boldsymbol{z}_{\boldsymbol{i}}\right|+\left|\boldsymbol{\alpha}_{\boldsymbol{i}}\right|$ which leads to $\left|\dot{\boldsymbol{q}}_{i}\right|<\boldsymbol{k}_{\boldsymbol{c} i}+\boldsymbol{c}_{1 i}+\overline{\boldsymbol{x}}_{\boldsymbol{d} 1 i}$.

The control objective on constraining the velocity variable has now been achieved. The control design will continue to obtain good tracking performance and bounding of the closed-loop signals.

Differentiation of $\boldsymbol{V}_{2}$ with respect to time gives,

$$
\dot{\boldsymbol{V}}_{2}=\dot{\boldsymbol{V}}_{1}+\boldsymbol{z}^{T}\left(\mathrm{~T}(\tau)+\boldsymbol{\Gamma}-\boldsymbol{C} \boldsymbol{\alpha}-\boldsymbol{G}-\boldsymbol{M} \dot{\boldsymbol{\alpha}}+\left(-\boldsymbol{C}+\frac{1}{2} \dot{\boldsymbol{M}}\right) \boldsymbol{z}\right)+\sum_{i=1}^{n} \frac{\pi z_{i} \dot{z}_{i}}{\boldsymbol{k}_{c i}{ }^{2}} \tan \left(\frac{\pi z_{i}{ }^{T} z_{i}}{2 \boldsymbol{k}_{c i}{ }^{2}}\right) \sec \left(\frac{\pi z_{i}{ }^{T} z_{i}}{2 \boldsymbol{k}_{c i}{ }^{2}}\right)
$$

By considering (11), Property 1 , and defining $\Lambda_{i}=\pi z_{i}{ }^{T} z_{i} / 2 \boldsymbol{k}_{c i}{ }^{2}$, and $\Upsilon_{i}=\tan \left(\Lambda_{i}\right) \sec \left(\Lambda_{i}\right)$, and the difference $\Delta \tau=\mathrm{T}(\tau)-\tau$, (13) can be rewritten as,

$$
\dot{V}_{2}=-\sum_{i=1}^{n} c_{1 i} \boldsymbol{k}_{1 i} \frac{\boldsymbol{e}_{i} \tanh \left(\boldsymbol{e}_{i}\right)}{\sqrt{1+\boldsymbol{e}_{i}^{2}}}+\sum_{i=1}^{n} \frac{\boldsymbol{k}_{1 i} \boldsymbol{e}_{i}}{\sqrt{1+\boldsymbol{e}_{i}^{2}}} z_{i}+z^{T}(\tau+\Delta \tau+\boldsymbol{\Gamma}-\boldsymbol{C} \boldsymbol{\alpha}-\boldsymbol{G}-\boldsymbol{M} \dot{\alpha})+2 \sum_{i=1}^{n} \Lambda_{i} \frac{\dot{z}_{i}}{z_{i}} \Upsilon_{i}
$$

Note that, in (13), and (14), $\dot{\alpha}$ is the derivation of the virtual control, $\alpha\left(x_{1}, x_{d}, \dot{x}_{d}\right)$, and is given by,

$$
\dot{\boldsymbol{\alpha}}=\frac{\partial \boldsymbol{\alpha}}{\partial \boldsymbol{x}_{1}} \boldsymbol{x}_{2}+\sum_{j=0}^{1} \frac{\partial \boldsymbol{\alpha}}{\partial \boldsymbol{x}_{d}^{(j)}} \boldsymbol{x}_{\boldsymbol{d}}^{(j+1)}
$$

In practical applications, due to uncertainties and unmeasurable factors, deriving the exact robotic dynamics is impossible. Thus, dynamic matrices $\boldsymbol{M}, \boldsymbol{C}$, and $\boldsymbol{G}$ are unknown and cannot be directly applied to design the control $\tau$. Also the amount of difference $\Delta \tau$ is unknown. Using the advantages of $N N$ in universal approximation and the learning capability in addition to structuring using a simple and fixed three-layer architecture, RBF is renowned as a reliable and effective approximator for the control of robotic systems [17, 32-35]. In this study, to compensate for the system uncertainties, the RBF NN is employed as,

$$
-C \boldsymbol{\alpha}-\boldsymbol{G}-M \dot{\alpha}+\Delta \tau=\omega^{* T} \boldsymbol{h}+\varepsilon .
$$

The RBF input $\boldsymbol{Z} \in \mathbf{R}^{n \times 4}$ is chosen as $\boldsymbol{Z}=\left[\boldsymbol{e}^{T}, z^{T}, \boldsymbol{\alpha}^{T}, \Lambda^{T}\right]$. In addition to handling the uncertainties in the control design, the modified Lyapunov function was considered to be,

$$
\boldsymbol{V}=\boldsymbol{V}_{2}+\frac{1}{2} \sum_{i=1}^{n} \tilde{\boldsymbol{\omega}}_{i}^{T} \Pi_{i}^{-1} \tilde{\boldsymbol{\omega}}_{i}+\frac{1}{2} \sum_{i=1}^{n} \tilde{\boldsymbol{\Gamma}}_{i}^{T} \tilde{\boldsymbol{\Gamma}}_{i}
$$

where $\tilde{\boldsymbol{\omega}}_{i}=\boldsymbol{\omega}^{*}-\hat{\boldsymbol{\omega}}, \tilde{\Gamma}=\Gamma-\hat{\Gamma}$, and $\Pi_{i}=\Pi_{i}{ }^{T}>0, \boldsymbol{i}=1,2, \ldots, \boldsymbol{n}$ is a gain matrix. The third term on the RHS of (17) is considered 
to cope with the unknown interaction forces, as in many human-robot interaction tasks interaction forces cannot be realized in practice.

In this section, by using RBF NN to approximate the unknown robot dynamics, and developing the proper adaptive laws and applying useful lemmas, the control $\tau$ was obtained without directly using any knowledge of the dynamic matrices $\boldsymbol{M}, \boldsymbol{C}$, and $\boldsymbol{G}$, and the interaction force, $\Gamma$.

Differentiation of $\boldsymbol{V}_{2}$ with respect to time leads to,

$$
\dot{\boldsymbol{V}}=\dot{\boldsymbol{V}}_{2}-\sum_{i=1}^{n} \tilde{\boldsymbol{\omega}}_{i}^{T} \Pi_{i}^{-1} \dot{\hat{\boldsymbol{\omega}}}_{i}-\sum_{i=1}^{n} \tilde{\boldsymbol{\Gamma}}_{i}^{T} \dot{\hat{\boldsymbol{\Gamma}}}_{i}
$$

The control $\tau$ can then be chosen as,

$$
\tau=-\hat{\boldsymbol{\omega}}^{T} \boldsymbol{h}-\hat{\boldsymbol{\Gamma}} \tanh \left(\frac{\boldsymbol{z}}{\varphi}\right)-c_{2} z-\sum_{i=1}^{n} \frac{\boldsymbol{k}_{1 i} \boldsymbol{e}_{i}}{\sqrt{1+\boldsymbol{e}_{i}^{2}}}-\sum_{i=1}^{n} \frac{\pi}{\boldsymbol{k}_{c i}{ }^{2}}\left(c_{3 i} z_{i}+a_{i}-\dot{\alpha}_{i}\right) \Upsilon_{i},
$$

and the adaption laws as,

$$
\begin{gathered}
\dot{\hat{\boldsymbol{\omega}}}_{i}=\Pi_{i}\left(\boldsymbol{h}_{i} z_{i}-\sigma_{\omega i} \hat{\boldsymbol{\omega}}_{i}\right) \\
\dot{\hat{\boldsymbol{\Gamma}}}_{i}=z_{i} \tanh \left(\frac{z_{i}}{\varphi}\right)-\sigma_{\gamma i} \hat{\boldsymbol{\Gamma}}_{i}
\end{gathered}
$$

where $c_{2}$ is the positive control gain matrix, and $\boldsymbol{c}_{3 i}, \boldsymbol{\sigma}_{\omega i}$, and $\sigma_{\boldsymbol{r}}$ for $\boldsymbol{i}=1,2, \ldots, \boldsymbol{n}$ are positive design constants. Note that the second terms of each adaption law in (20), and (21) are designed to improve the robustness of the system. Notice that without any modification of terms $\sigma_{\omega} \hat{\boldsymbol{\omega}}$, and $\sigma_{\gamma} \hat{\boldsymbol{\Gamma}}$, the estimation parameters for $\hat{\boldsymbol{\omega}}$, and $\hat{\boldsymbol{\Gamma}}$ will only be driven by the tracking error $\boldsymbol{z}$, which may decrease the robustness of the system.

Substituting control (19), adaption laws (20), and (21) in addition to (14) into (18), results in,

$$
\begin{aligned}
& \dot{V}=-\sum_{i=1}^{n} c_{1 i} \boldsymbol{k}_{1 i} \frac{\boldsymbol{e}_{i} \tanh \left(\boldsymbol{e}_{i}\right)}{\sqrt{1+\boldsymbol{e}_{i}^{2}}}+\sum_{i=1}^{n} \frac{\boldsymbol{k}_{1 i} \boldsymbol{e}_{i}}{\sqrt{1+\boldsymbol{e}_{i}^{2}}} z_{i}+z^{T}\left(-\hat{\omega}^{T} \boldsymbol{h}+\omega^{* T} \boldsymbol{h}+\boldsymbol{\varepsilon}+\boldsymbol{\Gamma}-\hat{\boldsymbol{\Gamma}} \tanh \left(\frac{z}{\varphi}\right)-c_{2} z\right)-\sum_{i=1}^{n} \tilde{\omega}_{i}^{T}\left(\boldsymbol{h}_{i} z_{i}-\sigma_{\omega i} \hat{\omega}_{i}\right) \\
& -\sum_{i=1}^{n} \tilde{\boldsymbol{\Gamma}}_{i}{ }^{T}\left(z_{i} \tanh \left(\frac{z_{i}}{\varphi}\right)-\sigma_{\gamma i} \hat{\boldsymbol{\Gamma}}_{i}\right)+\sum_{i=1}^{n} \frac{\pi}{\boldsymbol{k}_{c i}{ }^{2}} z_{i}{ }^{T} \dot{z}_{i} \Upsilon_{i}-\sum_{i=1}^{n} \frac{\boldsymbol{k}_{1 i} \boldsymbol{e}_{i}}{\sqrt{1+\boldsymbol{e}_{i}{ }^{2}}} z_{i}-\pi c_{3 i} \sum_{i=1}^{n} \frac{z_{i}{ }^{T} z_{i}}{\boldsymbol{k}_{c i}{ }^{2}} \Upsilon_{i} \\
& -\pi \sum_{i=1}^{n} \frac{z_{i}^{T}}{\boldsymbol{k}_{c i}{ }^{2}}\left(\boldsymbol{a}_{i}-\dot{\alpha}_{i}\right) \tan \left(\Lambda_{i}\right) \sec \left(\Lambda_{i}\right) \text {. }
\end{aligned}
$$

Applying Young's inequality, one has $\boldsymbol{z} \varepsilon \leq\|z\| \varepsilon_{M} \leq 1 / 2\|z\|^{2}+1 / 2 \varepsilon_{M}{ }^{2}$; then, since $\tilde{\boldsymbol{\omega}}_{i}^{T} \hat{\boldsymbol{\omega}}_{i}=\tilde{\boldsymbol{\omega}}_{i}^{T}\left(\boldsymbol{\omega}_{i}-\tilde{\boldsymbol{\omega}}_{i}\right)=\tilde{\boldsymbol{\omega}}_{i}{ }^{T} \boldsymbol{\omega}_{i}-\tilde{\boldsymbol{\omega}}_{i}^{T} \tilde{\boldsymbol{\omega}}_{i}$, it can be obtained that $\tilde{\omega}_{i}^{T} \omega_{i} \leq 1 / 2\left(\tilde{\omega}_{i}^{T} \tilde{\omega}_{i}+\omega_{i}{ }^{T} \omega_{i}\right)$, which gives $\quad \tilde{\omega}_{i}^{T} \hat{\omega}_{i} \leq-1 / 2\left(\tilde{\omega}_{i}{ }^{T} \tilde{\omega}_{i}-\omega_{i}{ }^{T} \omega_{i}\right)$. Similarly, $\tilde{\Gamma}_{i}^{T} \hat{\Gamma}_{i} \leq-1 / 2 \tilde{\Gamma}_{i}^{T} \tilde{\Gamma}_{i}+1 / 2 \Gamma_{i}^{T} \Gamma_{i}$, and further by applying Lemma 3, results in $\left|z_{i}\right| \Gamma_{i}-z_{i} \Gamma_{i} \tanh \left(z_{i} / \varphi\right) \leq \delta \Gamma_{i} \varphi$. Subsequently, noting that $\tanh (\boldsymbol{x}) \leq \boldsymbol{x}$ for all $\boldsymbol{x} \in \mathbf{R}$ and applying the above inequalities in (22) results in,

$$
\begin{aligned}
\dot{\boldsymbol{V}} \leq & -\sum_{i=1}^{n} \boldsymbol{c}_{1 i} \frac{\boldsymbol{e}_{i}{ }^{2}}{\sqrt{1+\boldsymbol{e}_{i}{ }^{2}}}-\frac{1}{2} z^{T}\left(2 c_{2}-\boldsymbol{I}\right) z-\pi \sum_{i=1}^{n} \boldsymbol{c}_{3 i} \frac{z_{i}{ }^{T} z_{i}}{\boldsymbol{k}_{c i}{ }^{2}} \Upsilon_{i}-\frac{1}{2} \sum_{i=1}^{n} \sigma_{\omega i} \tilde{\omega}_{i}^{T} \tilde{\omega}_{i}-\frac{1}{2} \sum_{i=1}^{n} \sigma_{\gamma i} \tilde{\Gamma}_{i}^{T} \tilde{\Gamma}_{i} \\
& +\frac{1}{2} \sum_{i=1}^{n} \sigma_{\omega i} \omega_{i}^{T} \omega_{i}+\frac{1}{2} \sum_{i=1}^{n} \sigma_{\gamma i} \Gamma_{i}^{T} \Gamma_{i}+\sum_{i=1}^{n} \delta \varphi \Gamma_{i}+\frac{1}{2} \varepsilon_{M}{ }^{2} \\
\leq & -\bar{\alpha} V_{2}+\bar{\beta},
\end{aligned}
$$

where, 


$$
\bar{\alpha}=\min \left(\frac{c_{1 i}}{\boldsymbol{k}_{1 i}}, \frac{\lambda_{\min }\left(2 c_{2}-I\right)}{\lambda_{\max }(\boldsymbol{M})}, c_{3 i}, \frac{\sigma_{\omega i}}{\lambda_{\max }\left(\Pi_{i}^{-1}\right)}, \sigma_{\gamma i}\right),
$$

and,

$$
\bar{\beta}=\frac{1}{2} \sum_{i=1}^{n} \sigma_{\omega i}\left\|\omega_{i}\right\|^{2}+\frac{1}{2} \sum_{i=1}^{n} \sigma_{\gamma i}\left\|\Gamma_{i}\right\|^{2}+\sum_{i=1}^{n} \delta \varphi \Gamma_{i}+\frac{1}{2} \varepsilon_{M}^{2}
$$

Note that following the explanation in the paper to choose the design parameters and choosing $c_{2}$ that satisfies $\boldsymbol{c}_{2 i}>1 / 2$ yields $\bar{\alpha}, \overline{\boldsymbol{\beta}}>0$. In addition, to satisfying the conditions defined by Lemma 2 , the constants $\boldsymbol{c}_{3 i}$ should be selected such that $\boldsymbol{c}_{3 i} \geq 1$. Also, it is clear from the existence of $\overline{\boldsymbol{\beta}} \neq 0$ that the system just achieves the stability, but it could not achieve the exponential stability. The summary of the main outcomes can then be written in the following theorem.

Theorem 1: Consider the robot dynamics (5) satisfying Assumptions 1, and 2, the virtual control (10), the closed-loop control law (19) and adaptive laws (20), and (21), with the initial set defined by $\Omega_{i}=\left\{\dot{\boldsymbol{q}}_{i}, \boldsymbol{i}=1, \ldots, \boldsymbol{n}|| \dot{\boldsymbol{q}}_{\boldsymbol{i}}(0) \mid<\boldsymbol{k}_{\boldsymbol{v} i}\right\}$, and let $Z_{i}=\max _{x \in \Omega_{0}}\left|z_{i}\left(\boldsymbol{x}_{1 i}, \boldsymbol{x}_{d i}(0), \dot{\boldsymbol{x}}_{d i}(0)\right)\right|, \boldsymbol{i}=1,2, \ldots, \boldsymbol{n}$. Let $\left|\boldsymbol{\alpha}_{i}\right| \leq \bar{\alpha}_{i}$ with $\overline{\boldsymbol{\alpha}}_{i}=\boldsymbol{c}_{1 i}+\overline{\boldsymbol{x}}_{d 1 i}$, and the velocity constraint defined by $\left|\dot{\boldsymbol{q}}_{i}\right|<\boldsymbol{k}_{v i}$, with the given $\boldsymbol{k}_{\boldsymbol{v} i}$ for $\boldsymbol{i}=1,2, \ldots, \boldsymbol{n}$. If there exist positive constants $\mathfrak{I}=\left[\boldsymbol{a}_{\boldsymbol{i}}, \boldsymbol{b}_{\boldsymbol{i}}, \boldsymbol{c}_{1 i}, \boldsymbol{k}_{c i}\right]^{T}, \boldsymbol{i}=1,2, \ldots, \boldsymbol{n}$, that satisfy the following feasibility conditions,

$$
\begin{aligned}
& \boldsymbol{k}_{v i}>\overline{\boldsymbol{\alpha}}_{i}+\boldsymbol{k}_{c i} \\
& \boldsymbol{k}_{c i}>Z_{i}(\Im), \boldsymbol{i}=1, \ldots, \boldsymbol{n},
\end{aligned}
$$

then, the following properties hold.

i. The position tracking error, e, converges to a small neighborhood of zero, with the design parameters being properly chosen.

ii. The velocity variable $\dot{\boldsymbol{q}}_{\boldsymbol{i}}(\boldsymbol{t})$ remains, for all $\boldsymbol{t}>0$, in the open constraint set $\Omega_{v}=\left\{\dot{\boldsymbol{q}}_{i}, \boldsymbol{i}=1, \ldots, \boldsymbol{n}|| \dot{\boldsymbol{q}}_{\boldsymbol{i}} \mid<\boldsymbol{k}_{\boldsymbol{v}}(\boldsymbol{t})\right\}$.

iii. All closed-loop signals are bounded.

Proof:

$i$. Denote $\boldsymbol{\zeta}=\overline{\boldsymbol{\beta}} / \overline{\boldsymbol{\alpha}}>0$, then (23) satisfies,

$$
0 \leq \boldsymbol{V}(\boldsymbol{t}) \leq \boldsymbol{\zeta}+\boldsymbol{V}(0) \exp (-\overline{\boldsymbol{\alpha}} \boldsymbol{t}), \forall \boldsymbol{t}>0,
$$

which implies that the Lyapunov function $\boldsymbol{V}(\boldsymbol{t})$ is bounded. From (27), one can obtain $\boldsymbol{k}_{1 i}\left(\sqrt{1+\boldsymbol{e}_{i}^{2}}-1\right)<\boldsymbol{\zeta}+\boldsymbol{V}(0) \exp (-\overline{\boldsymbol{\alpha}} \boldsymbol{t})$, for $\boldsymbol{i}=1, \ldots, \boldsymbol{n}$. Further, by applying some manipulations it is obtained that $\boldsymbol{e}_{i}<\boldsymbol{\zeta}^{2}\left(1+2 \boldsymbol{k}_{1 i} / \boldsymbol{\zeta}\right) / \boldsymbol{k}_{1 i}{ }^{2}+\boldsymbol{V}(0)\left(\exp (-2 \overline{\boldsymbol{\alpha}} \boldsymbol{t})-2\left(\boldsymbol{\zeta}+\boldsymbol{k}_{1 i}\right) \exp (-\bar{\alpha} \boldsymbol{t})\right) / \boldsymbol{k}_{1 i}{ }^{2}$ which implies that, given $\boldsymbol{v}_{\boldsymbol{i}}>\boldsymbol{\zeta}^{2}\left(1+2 \boldsymbol{k}_{1 i} / \boldsymbol{\zeta}\right) / \boldsymbol{k}_{1 i}{ }^{2}$, there exist $\boldsymbol{T}>0$ such that,

$$
\left|\boldsymbol{e}_{i}\right|<\boldsymbol{v}_{i}, \forall \boldsymbol{t} \geq \boldsymbol{T} \text { and } \boldsymbol{i}=1, \ldots, \boldsymbol{n},
$$

where $v_{i}$ is the size of a small residual set showing the convergence property of the error.

ii. First, from the satisfaction of the second condition in (26), i.e., $\boldsymbol{k}_{c i}>Z_{i}$, one can obtain $\left|z_{i}(\boldsymbol{t})\right|<\boldsymbol{k}_{c i}$, for all $\boldsymbol{t}>0$. In addition, since $\dot{\boldsymbol{q}}_{i}=\boldsymbol{x}_{2 i}=\boldsymbol{z}_{i}+\alpha_{i}$, and $\left|\boldsymbol{\alpha}_{i}\right| \leq \overline{\boldsymbol{\alpha}}_{i}$, and noting that $\bar{\alpha}_{i}+\boldsymbol{k}_{c i}<\boldsymbol{k}_{v i}$, according to the first condition of (26), it can be 
concluded that $\left|\dot{\boldsymbol{q}}_{i}\right|<\boldsymbol{k}_{v i}$. Accordingly, the control objective on the remaining joint velocity $\dot{\boldsymbol{q}}(\boldsymbol{t})$ within the constrained region $\Omega_{v}$, is satisfied for all $\boldsymbol{t}>0$.

iii. From $i$. it can be seen that $\boldsymbol{e}$ is bounded and using Assumption 2 it is clear that $\boldsymbol{x}_{1}$ is bounded. Similar to $i$, it can be obtained that $\left|\boldsymbol{x}_{2}\right| \leq\left(2 \boldsymbol{\zeta}+2 \boldsymbol{V}(0) \exp (-\bar{\alpha} \boldsymbol{t}) / \boldsymbol{\lambda}_{\min }(\boldsymbol{M})\right)^{1 / 2}$, and since the variable transformation $\boldsymbol{z}$ is bounded, as shown in ii., then $\alpha$ becomes bounded. Further, it can be easily shown from Lemma 4 , that $\dot{\boldsymbol{x}}_{2}=\boldsymbol{a}$, and $\dot{\boldsymbol{\alpha}}$ also remains bounded. On the other hand, as the Lyapunov function $\boldsymbol{V}$ is bounded, then $\hat{\boldsymbol{\omega}}$ and $\hat{\Gamma}$ are proven to be bounded; in addition, since $\boldsymbol{h}\left(\boldsymbol{Z}_{\boldsymbol{i}}\right)$ is bounded as $\boldsymbol{h}\left(\boldsymbol{Z}_{\boldsymbol{i}}\right) \leq \boldsymbol{\varpi}_{\boldsymbol{i}}$ with finite constant $\boldsymbol{\varpi}_{\boldsymbol{i}}>0$; then, using (19), the control input $\boldsymbol{u}$ can be shown to be bounded, as well. Therefore, from (8), (12), (17) and (27) it is confirmed that all closed-loop signals in the closed-loop system remain bounded.

Remark 3: As shown in (28) by reducing $\boldsymbol{v}$, the convergence value of the steady state error can be reduced. It can be done by reducing $\zeta=\overline{\boldsymbol{\beta}} / \overline{\boldsymbol{\alpha}}$, and thus, increasing $\overline{\boldsymbol{\alpha}}$, or reducing $\overline{\boldsymbol{\beta}}$. However, choosing large $\boldsymbol{c}_{1}, \boldsymbol{c}_{2}$, or $\boldsymbol{c}_{3}$ in order to obtain larger $\overline{\boldsymbol{\alpha}}$ may lead to the excitation of unmodeled dynamics as a result of increasing the motor input voltage. On the other hand, choosing small $\sigma_{\omega}$, and $\sigma_{\gamma}$ to obtain smaller $\overline{\boldsymbol{\beta}}$, may lead to large NN estimation weights, or reduced system robustness to external forces. Accordingly, to choose the control parameters, the balance between tracking and system performances should be considered.

\section{Feasibility Check}

In this section, we investigate the validity of the proposed control scheme by checking the feasibility conditions. By that means we address conditions for existence of a set of design parameters such that efficient tracking is achieved without violating the velocity constraints. Specifically, we formulate and offline solve a static nonlinear constrained optimization problem, in terms of the design parameters, prior to actual implementation of the control scheme.

The feasibility conditions are expressed as sufficient conditions defined by (26) in Theorem 1 . The condition is dependent on the design parameters, the initial conditions, and the velocity constraints. Accordingly, finding a set of design parameters that can satisfy (26), will lead to the feasible control using such parameters.

To do this, we check if a solution exists,

$$
\mathfrak{J}=\left[a_{i}, b_{i}, c_{1 i}, k_{c i}\right]^{T},
$$

for the following optimization problem:

Minimize the objective function

$$
\boldsymbol{J}(\mathfrak{J})=-\left(\gamma_{1} \sum_{i=1}^{n} \boldsymbol{c}_{1 i}+\gamma_{2} \sum_{i=1}^{n} \boldsymbol{k}_{c i}+\sum_{i=1}^{n}\left(\boldsymbol{b}_{i}-\boldsymbol{a}_{i}\right)\right),
$$

subject to

$$
\begin{aligned}
& \boldsymbol{k}_{v i}>\overline{\boldsymbol{\alpha}}_{\boldsymbol{i}}+\boldsymbol{k}_{c i} \\
& \boldsymbol{k}_{c i}>\mathrm{Z}_{i}(\mathfrak{I}) \\
& -\boldsymbol{k}_{\boldsymbol{b i}}<\boldsymbol{a}_{\boldsymbol{i}}<\boldsymbol{b}_{\boldsymbol{i}}<\boldsymbol{k}_{\boldsymbol{b i}} \\
& \boldsymbol{c}_{1 i}>0, \quad \boldsymbol{i}=1, \ldots, \boldsymbol{n},
\end{aligned}
$$

where $\gamma_{1}$, and $\gamma_{2}$ are positive weighing constants. If a solution $\mathfrak{I}^{*}$ exists, then conditions (26) in Theorem 1 are satisfied, then the proposed control (19) with $\mathfrak{I}=\mathfrak{J}^{*}$ is feasible to guarantee tracking for a robot system (5) with velocity constraint.

\section{Controller modification for asymmetric and time-varying constraints}

Motivated by several practical robotic applications which are subject to time-varying constraints [36], in this section, we 
present time-varying constrained control by modifying the presented controller in Section 3. The controller is also further developed to include asymmetric constraints. By that means, more flexible constraints can be modeled for various practical transitions. Furthermore, the required initial conditions can be relaxed effectively on the starting values of the joint movement. To this end, we first state our new control objective and then introduce a new control assumption as follows,

The control objective is to design an adaptive controller for the robot dynamic system given by (5) under the existence of velocity constraints where $\dot{\boldsymbol{q}}(\boldsymbol{t})$ remains in the constrained region $\Omega_{v m}=\left\{\dot{\boldsymbol{q}}_{i} \in \mathbf{R}, \boldsymbol{i}=1, \ldots, \boldsymbol{n} \mid \underline{\boldsymbol{k}}_{v i}(\boldsymbol{t})<\dot{\boldsymbol{q}}_{i}(\boldsymbol{t})<\overline{\boldsymbol{k}}_{v i}(\boldsymbol{t}), \boldsymbol{t} \geq 0\right\}$, i.e., $\dot{\boldsymbol{q}}(\boldsymbol{t}) \in \Omega_{v m}, \boldsymbol{t}>0$, provided $\dot{\boldsymbol{q}}(0) \in \Omega_{v m}$, with $\overline{\boldsymbol{k}}_{v i}(\boldsymbol{t})$ and $\underline{\boldsymbol{k}}_{v i}(\boldsymbol{t})$ being bounded pre-specified functions such that $\overline{\boldsymbol{k}}_{v i}(\boldsymbol{t})>\underline{\boldsymbol{k}}_{v i}(\boldsymbol{t}) \forall \boldsymbol{t} \in \mathbf{R}^{+}$, such that all the signals in the closed-loop system remain bounded and robot joint positions follow the given desired trajectories $\boldsymbol{x}_{d}(t)=\left[\boldsymbol{x}_{d 1}, \boldsymbol{x}_{d 2}, \ldots, \boldsymbol{x}_{d n}\right]^{T}$ as closely as possible, i.e., $\lim _{t \rightarrow \infty}\left|\boldsymbol{q}_{i}(\boldsymbol{t})-\boldsymbol{x}_{d i}(\boldsymbol{t})\right|=\boldsymbol{t}_{i}$ with $\boldsymbol{t}_{i}$ being a small positive constant.

Assumption 3 [31]: There exist positive constants $\overline{\boldsymbol{K}}_{v i}$, and $\underline{\boldsymbol{K}}_{v i}$ such that $\left|\dot{\overline{\boldsymbol{k}}}_{v i}(\boldsymbol{t})\right| \leq \overline{\boldsymbol{K}}_{v i}, \quad\left|\dot{\boldsymbol{k}}_{v i}(\boldsymbol{t})\right| \geq \underline{\boldsymbol{K}}_{v i}$, for $\boldsymbol{i}=1, \ldots, \boldsymbol{n}$, $\forall \boldsymbol{t}>0$. Also, there exist positive constants $\overline{\boldsymbol{k}}_{m i}, \underline{\boldsymbol{k}}_{m i}, \overline{\boldsymbol{k}}_{n i}$ and $\underline{\boldsymbol{k}}_{n i}$, such that $\underline{\boldsymbol{k}}_{m i}<\overline{\boldsymbol{k}}_{z i}(\boldsymbol{t})<\overline{\boldsymbol{k}}_{m i}$ and $\underline{\boldsymbol{k}}_{n i}<\underline{\boldsymbol{k}}_{z i}(\boldsymbol{t})<\overline{\boldsymbol{k}}_{n i}$, $\boldsymbol{i}=1, \ldots, \boldsymbol{n}, \forall \boldsymbol{t} \geq 0 \quad$ where $\quad \overline{\boldsymbol{k}}_{z i}(\boldsymbol{t})$ and $\underline{\boldsymbol{k}}_{z i}(\boldsymbol{t})$ are time-varying velocity tracking error barriers, defined by $\underline{\boldsymbol{k}}_{z i}(\boldsymbol{t})=\underline{\boldsymbol{k}}_{v i}(\boldsymbol{t})-\alpha_{i}(\boldsymbol{t})$ and $\overline{\boldsymbol{k}}_{z i}(\boldsymbol{t})=\overline{\boldsymbol{k}}_{v i}(\boldsymbol{t})-\alpha_{i}(\boldsymbol{t})$.

Remark 4: A number of lower or upper bounds are defined by Assumption 3. These bounds will be used to develop the control algorithm and stability analysis. Nevertheless, these parameters, although existing, will not be involved in designing the control. Accordingly, actual estimation of them will not be required in setting up and implementing the control scheme.

Now, we are ready to state an asymmetric and time-varying constrained control scheme.

Consider the following modified Lyapunov function based on the asymmetric and time-varying sBLF,

$$
\boldsymbol{V}_{2 m}=\boldsymbol{V}_{1}+\frac{1}{2} \boldsymbol{z}^{T} \boldsymbol{M} \boldsymbol{z}+\sum_{i=1}^{n} \sec \left(\frac{\pi z_{i}^{T} z_{i}}{2 \boldsymbol{k}_{w i}{ }^{2}}\right)-1
$$

where $\boldsymbol{k}_{w i}(\boldsymbol{t})=\overline{\boldsymbol{k}}_{z i}(\boldsymbol{t})$, if $\boldsymbol{e}_{1 i}(\boldsymbol{t})>0$, otherwise $\boldsymbol{k}_{w i}(\boldsymbol{t})=\underline{\boldsymbol{k}}_{z i}(\boldsymbol{t})$. Differentiation of $\boldsymbol{V}_{2 m}$ with respect to time, and considering (11), Property 1 , and defining $\Lambda_{m i}=\pi z_{i}{ }^{T} z_{i} / 2 \boldsymbol{k}_{w i}{ }^{2}, \Upsilon_{m i}=\tan \left(\frac{\pi z_{i}{ }^{T} z_{i}}{2 \boldsymbol{k}_{w i}{ }^{2}}\right) \sec \left(\frac{\pi z_{i}{ }^{T} z_{i}}{2 \boldsymbol{k}_{w i}{ }^{2}}\right)$ and gives,

$$
\dot{V}_{2 m}=-\sum_{i=1}^{n} c_{1 i} \boldsymbol{k}_{1 i} \frac{\boldsymbol{e}_{i} \tanh \left(\boldsymbol{e}_{i}\right)}{\sqrt{1+\boldsymbol{e}_{i}^{2}}}+\sum_{i=1}^{n} \frac{\boldsymbol{k}_{1 i} \boldsymbol{e}_{i}}{\sqrt{1+\boldsymbol{e}_{i}^{2}}} z_{i}+\boldsymbol{z}^{T}(\tau+\Delta \tau+\boldsymbol{\Gamma}-\boldsymbol{C} \boldsymbol{\alpha}-\boldsymbol{G}-\boldsymbol{M} \dot{\alpha})+\sum_{i=1}^{n} 2 \Lambda_{m i} \frac{\dot{\boldsymbol{z}}_{i}}{z_{i}} \Upsilon_{m i}-\sum_{i=1}^{n} 2 \Lambda_{m i} \frac{\dot{\boldsymbol{k}}_{w i}}{\boldsymbol{k}_{w i}} \Upsilon_{m i} .
$$

Then, considering uncertain terms, we can modify the Lyapunov function at (30), as $\boldsymbol{V}_{m}=\boldsymbol{V}_{2 m}+0.5 \sum_{i=1}^{n} \tilde{\omega}_{i}^{T} \Pi_{i}^{-1} \tilde{\omega}_{i}+0.5 \sum_{i=1}^{n} \tilde{\boldsymbol{\Gamma}}_{i}^{T} \tilde{\boldsymbol{\Gamma}}_{i}$. We choose the modified control as,

$$
\tau=-\hat{\boldsymbol{\omega}}^{T} \boldsymbol{h}-\hat{\boldsymbol{\Gamma}} \tanh \left(\frac{\boldsymbol{z}}{\boldsymbol{\varphi}}\right)-\boldsymbol{c}_{2} z-\sum_{i=1}^{n} \frac{\boldsymbol{k}_{1 i} \boldsymbol{e}_{i}}{\sqrt{1+\boldsymbol{e}_{i}^{2}}}+\sum_{i=1}^{n} \pi \frac{z_{i}}{\boldsymbol{k}_{w i}^{2}}\left(-c_{3 i}-\frac{\left(\boldsymbol{a}_{i}-\dot{\alpha}_{i}\right)}{z_{i}}+\frac{\dot{\boldsymbol{k}}_{w i}}{\boldsymbol{k}_{w i}}\right) \Upsilon_{m i}
$$

which with the same adaptive laws as in (20), and (21), and applying the same calculation as in Section 3, we can obtain $\dot{V}_{m} \leq-\bar{\alpha} V_{m}+\bar{\beta}$ with the same $\bar{\alpha}$ and $\bar{\beta}$ as in (24), and (25), respectively.

Before presenting the theorem for the asymmetric and time-varying velocity constrained control systems, we note that for the simplicity, and also avoiding repetition, we do not consider the feasibility checking for this case. However, the feasibility checking is similar to those presented in the time-invariant case. 
Theorem 2: Consider the robot dynamics (5) satisfying Assumptions 1-3, the virtual control (10), the closed-loop control law (32) and adaptive laws (20), and (21), with the initial set defined by $\Omega_{i m}=\left\{\dot{\boldsymbol{q}}_{\boldsymbol{i}} \in \mathbf{R}, \boldsymbol{i}=1, \ldots, \boldsymbol{n} \mid \underline{\boldsymbol{k}}_{\boldsymbol{v} \boldsymbol{i}}(0)<\dot{\boldsymbol{q}}_{\boldsymbol{i}}(0)<\overline{\boldsymbol{k}}_{\boldsymbol{v} i}(0)\right\}$, under the velocity constraint defined by $\underline{\boldsymbol{k}}_{\boldsymbol{v} i}(\boldsymbol{t})<\dot{\boldsymbol{q}}_{\boldsymbol{i}}<\overline{\boldsymbol{k}}_{\boldsymbol{v} \boldsymbol{i}}(\boldsymbol{t})$ for $\boldsymbol{i}=1,2, \ldots, \boldsymbol{n}$, and assume that there exists a sufficiently large compact $\Omega_{z}$, such that $\boldsymbol{Z}_{\boldsymbol{j}} \in \Omega_{z}, \forall \boldsymbol{t} \geq 0$, for $\boldsymbol{j}=1,2, \ldots, \boldsymbol{r}$. Then, the following properties hold.

$i$. The position tracking error, e, converges to a small neighborhood of zero, with the design parameters being properly chosen.

ii. The velocity variable $\dot{\boldsymbol{q}}_{i}(\boldsymbol{t})$ remains, for all $\boldsymbol{t}>0$, in the open constraint set $\Omega_{v}=\left\{\dot{\boldsymbol{q}}_{i}, \boldsymbol{i}=1, \ldots, \boldsymbol{n} \mid \underline{\boldsymbol{k}}_{v i}(\boldsymbol{t})<\dot{\boldsymbol{q}}_{\boldsymbol{i}}<\overline{\boldsymbol{k}}_{v \boldsymbol{i}}(\boldsymbol{t})\right\}$.

iii. All closed-loop signals are bounded.

Proof: The proof is similar to the proof of Theorem 1.

\section{Examples of simulation}

To illustrate the effectiveness of the developed control scheme, simulation studies were performed on a simple robot manipulator with two revolute joints in the vertical plane. The section includes three case studies. The first case study illustrates the tracking performance of the proposed control without violating constraints. The second case study highlights the ability of the presented method to cope with time-varying constrained sets while relaxing different initial conditions. In the third case study the presented sBLF is compared with the available logarithm type BLF.

\subsection{First Case Study}

The control objective of this simulation study was to track the desired joint trajectories as $\boldsymbol{x}_{d}=[\cos (\boldsymbol{t}),-\cos (\boldsymbol{t})]^{T}$ as closely as possible, while satisfying the velocity constraints by $\left|\dot{\boldsymbol{q}}_{\boldsymbol{i}}\right|<\boldsymbol{k}_{\boldsymbol{v}}$, with $\boldsymbol{k}_{\boldsymbol{v}}=3$, for $\boldsymbol{i}=1,2$, and guaranteeing the boundedness of other closed-loop system signals. The time period of the simulation covered $\boldsymbol{t}=10$ second. In this simulation we consider the control design presented in Section 3 with the feasibility checking that was presented in Section 4 . The initial condition of the robot was given by $\boldsymbol{q}(0)=[\pi / 4,-\pi / 4]^{T}, \dot{\boldsymbol{q}}(0)=[0,0]^{T}$, and the force vector $\Gamma$ was chosen as $\Gamma=[0.4 \sin (\boldsymbol{t}),-0.3 \cos (\boldsymbol{t})]^{T}$. Also, physical robot parameters were chosen as mass of link $1 \boldsymbol{m}_{1}=4 \mathrm{~kg}$, mass of link $2 \boldsymbol{m}_{2}=2 \mathrm{~kg}$, length of link $1 \boldsymbol{l}_{1}=1 \mathrm{~m}$, length of link $2 \boldsymbol{l}_{2}=0.5 \mathrm{~m}$, inertia of link $1 \boldsymbol{I}_{1}=0.2 \mathrm{kgm}^{2}$, and inertia of link $2 \boldsymbol{I}_{2}=0.2 \mathrm{kgm}^{2}$.

To do the simulation study, the unknown system model was considered and to approximate uncertainties a RBF NN with fifty nodes on each hidden layer with the centers $\vartheta_{i}$ evenly distributed in the span of input space $[-1.5,1.5]$, and widths of $\psi=10$ were chosen. The starting points of NN weights and adapting laws were chosen as $\hat{\omega}_{i}(0)=\Gamma_{i}(0)=0$, with control parameters chosen to be $\sigma_{w i}=\sigma_{y i}=0.05, \Pi_{i}=200, \boldsymbol{k}_{1 i}=1$, and $\varphi_{i}=0.5$, for $i=1,2$. The saturation parameter values were set to $\mathrm{T}_{\max }=[50,50]^{T} ;$ In this study, the Matlab routine fmincon.m was utilized to perform the feasibility check. Gains were chosen as $\gamma_{1}=3$, and $\gamma_{2}=1$, then by solving the constrained optimization problem as in Section 4 , we obtain $\boldsymbol{c}_{11}^{*}=\boldsymbol{c}_{12}^{*}=1.0704$, $\boldsymbol{k}_{c 1}^{*}=\boldsymbol{k}_{c 2}^{*}=1.0820, \boldsymbol{a}_{1}^{*}=\boldsymbol{a}_{2}^{*}=-1.2931, \boldsymbol{b}_{1}^{*}=\boldsymbol{b}_{2}^{*}=1.2931$ and choose $\boldsymbol{c}_{2 \boldsymbol{i}}=\boldsymbol{c}_{3 \boldsymbol{i}}=1$ for $\boldsymbol{i}=1,2$. The simulation figures are listed in Figs. 1-6. 

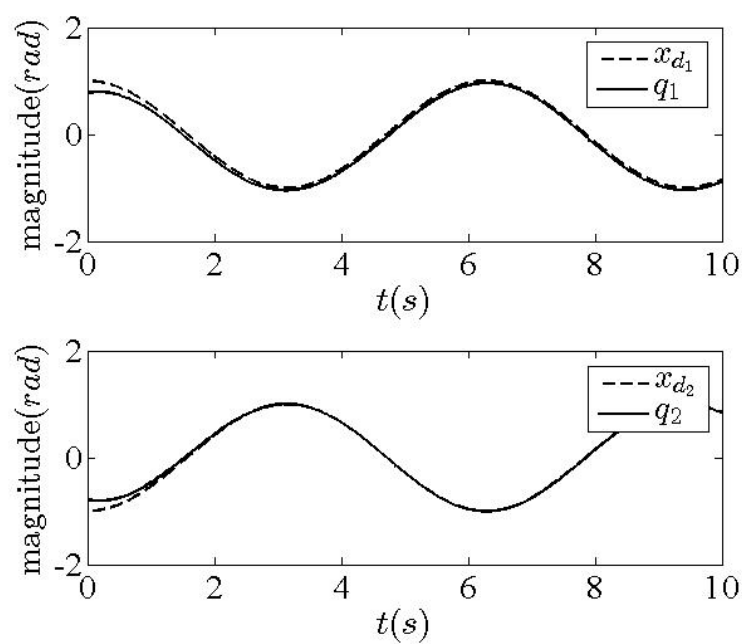

Fig. 1. Desired trajectory $\boldsymbol{x}_{\boldsymbol{d}}$ and actual trajectory $\boldsymbol{q}$ of joint positions for the uncertain control case.

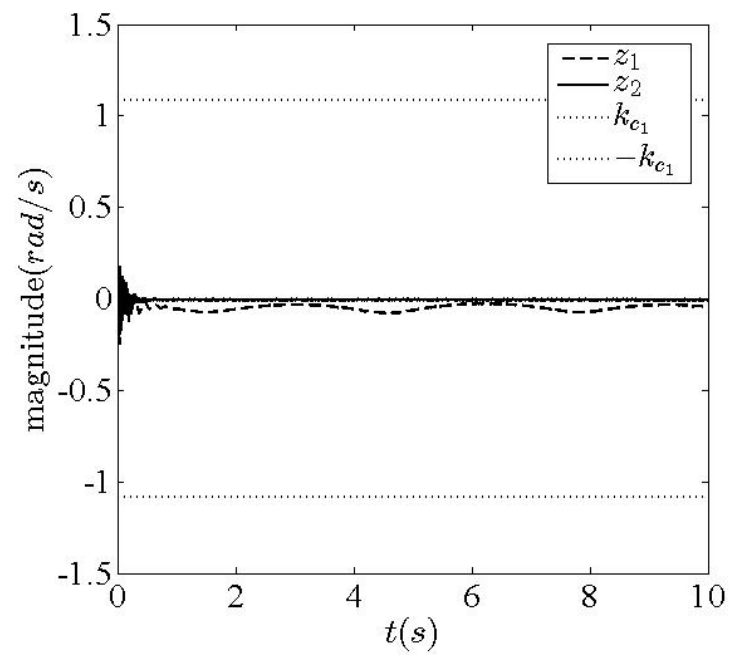

Fig. 3. Trajectory of error function $\boldsymbol{z}$, with constraints $\boldsymbol{k}_{\boldsymbol{c}}$, and $-\boldsymbol{k}_{\boldsymbol{c}}$ for the uncertain case.

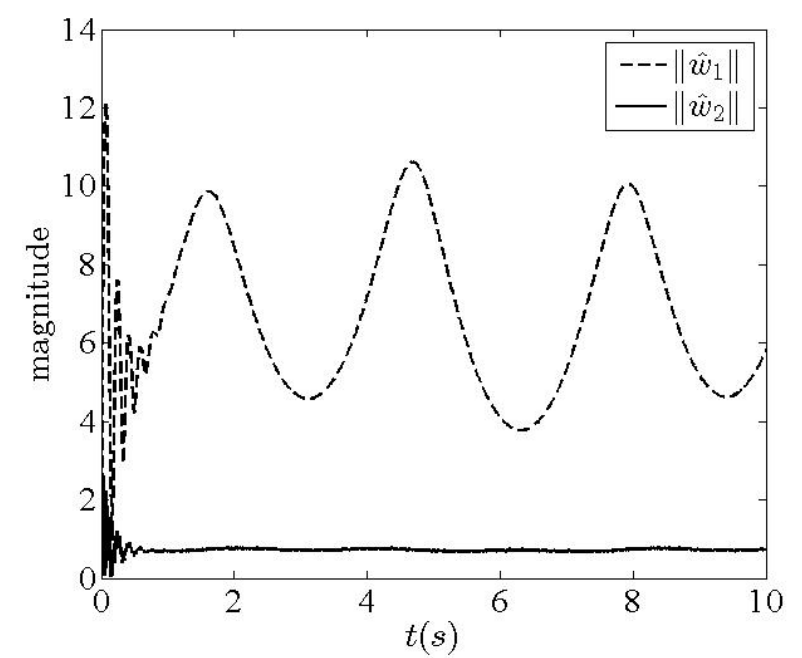

Fig. 5. Norms of RBF NN weights under the proposed control.
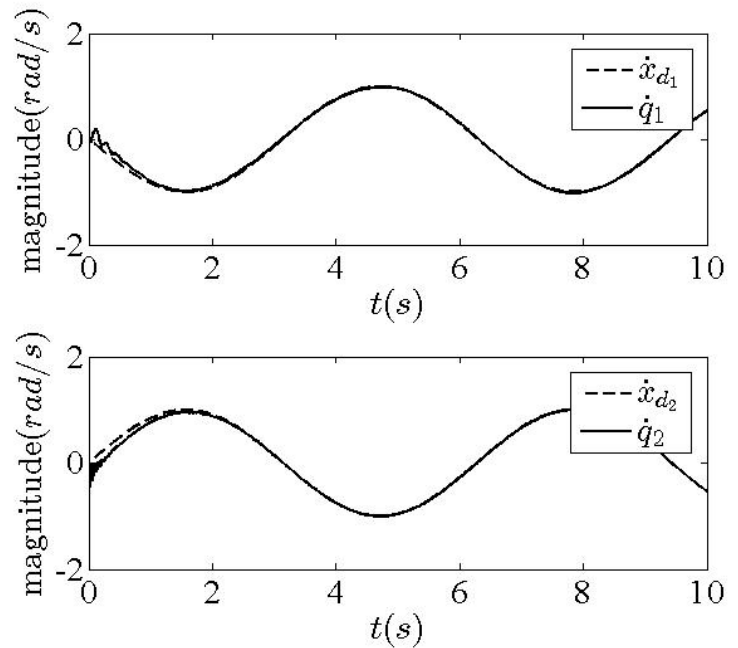

Fig. 2. Desired trajectory $\dot{\boldsymbol{x}}_{\boldsymbol{d}}$ and actual trajectory $\dot{\boldsymbol{q}}$ of joint velocities for the uncertain control case.

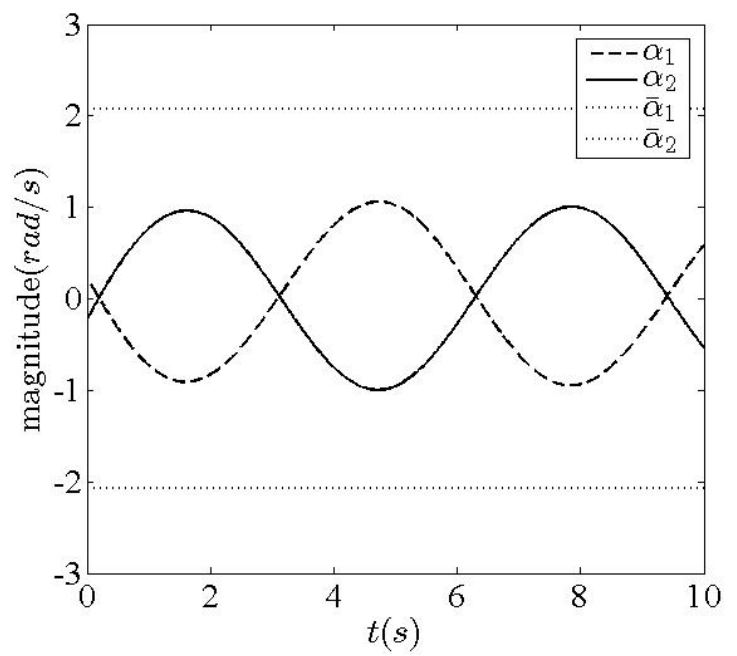

Fig. 4. Trajectory of virtual control $\alpha$, with constraint $\overline{\boldsymbol{a}}$ for the uncertain case.

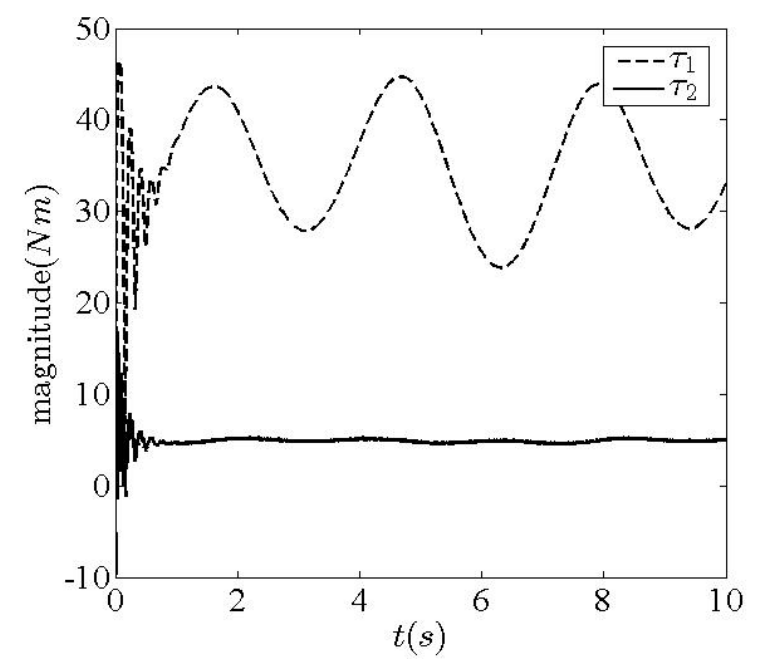

Fig. 6. Trajectory of control input $\tau$ for the uncertain control case. 
Figs. 1-3 show the tracking performance of the controller. Figs. 1, and 2 demonstrate that joint position and joint velocity effectively track their references, respectively. The boundedness of error function $\boldsymbol{z}$, and virtual control $\boldsymbol{\alpha}$ are demonstrated in Fig. 3, and Fig. 4, respectively. As shown in these figures, the values of $z$, and $\alpha$ never violate their predefined constraints. The former is because of the advantages of the introduced sBLF as discussed in the Introduction and in the Section II. A, and the latter is due to choosing bounded virtual control using the tan-hyperbolic function, and further boundedness of $\dot{\boldsymbol{x}}_{\boldsymbol{d}}$. The estimated NN weights in the sense of two-norm are shown on Fig. 5, which demonstrates that the norms are bounded and eventually converge to certain values. Figure 6 . shows that the control signals are saturated, while the performance of tracking is satisfactory. The figures show that the presented constrained adaptive NN control can satisfy our objectives on the tracking, constraint violation, and that the boundedness of the closed-loop signals are achieved.

\subsection{Second case study}

In this case study, the simulation was performed to highlight the effectiveness of the proposed method to provide constrained behavior where variables are growing close to their bounds. To this end, we render smaller ranges for the constrained error sets. Also, we use the asymmetric and time-varying constrained control design as presented in Section 5 . The velocity constraints are written in the form,

$$
\begin{aligned}
& \overline{\boldsymbol{k}}_{v i}=\boldsymbol{a}_{u i} \exp (-\boldsymbol{t})+\boldsymbol{\alpha}_{i}+\boldsymbol{a}_{o i}, \\
& \underline{\boldsymbol{k}}_{v i}=-\boldsymbol{a}_{l i} \exp (-\boldsymbol{t})+\boldsymbol{\alpha}_{i}-\boldsymbol{a}_{\boldsymbol{o}},
\end{aligned}
$$

for $\boldsymbol{i}=1,2$, where $\boldsymbol{a}_{\boldsymbol{o} i}$ denotes the required constraint values of the $\boldsymbol{i}^{\text {th }}$ joint, and $\boldsymbol{a}_{\boldsymbol{u}}$, and $\boldsymbol{a}_{l i}$ can be defined according to the initial conditions. Accordingly, using the above asymmetric time-varying constraints, the constraint boundaries can cover any initial conditions, and they then exponentially tend to be close to $\alpha_{i}$ as $\lim _{\boldsymbol{t} \rightarrow 0} \boldsymbol{a}_{\boldsymbol{u} i} \exp (-\boldsymbol{t})=\boldsymbol{a}_{\boldsymbol{u} i}$, and $\lim _{\boldsymbol{t} \rightarrow 0} \boldsymbol{a}_{l i} \exp (-\boldsymbol{t})=\boldsymbol{a}_{l i}$, and $\lim _{t \rightarrow \infty} \boldsymbol{a}_{\boldsymbol{u}} \exp (-\boldsymbol{t})=\lim _{\boldsymbol{t} \rightarrow \infty} \boldsymbol{a}_{l i} \exp (-\boldsymbol{t})=0$.

In this simulation, the magnitude of constraint parameters $\boldsymbol{a}_{0 i}$ are decreased to $\boldsymbol{a}_{\boldsymbol{}}=\boldsymbol{a}_{\boldsymbol{0} 2}=0.2$. Also, other corresponding parameters are considered as $\boldsymbol{a}_{1 u}=\boldsymbol{a}_{1 l}=1.4, \quad \boldsymbol{a}_{2 u}=1.4$, and $\boldsymbol{a}_{2 l}=2$. The desired trajectory is considered as $\boldsymbol{x}_{\boldsymbol{d}}=[0.5 \sin (\boldsymbol{t}),-0.5 \sin (\boldsymbol{t})]^{T}$, and the position of the robot initial joint condition is selected $\boldsymbol{q}(0)=[\boldsymbol{\pi} / 6,-\boldsymbol{\pi} / 6]^{T}$. The control parameters are selected as $\boldsymbol{c}_{1 i}=1, \quad \boldsymbol{c}_{2 i}=\boldsymbol{k}_{1 i}=500, \boldsymbol{c}_{3 i}=100$ for $\boldsymbol{i}=1,2$. In addition, for better illustration of the effects of the BLF terms on bounding of the error signals, the saturation bounds on controls are removed. Other simulation parameters are the same for the first simulation. The simulation results are illustrated in Figs. 7 - 9.
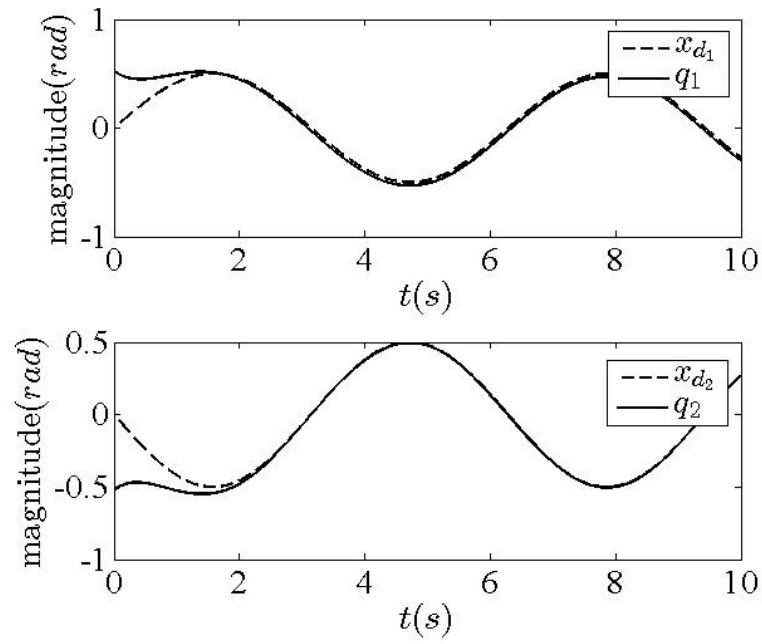

Fig. 7. Desired trajectory $\boldsymbol{x}_{\boldsymbol{d}}$ and actual trajectory $\boldsymbol{q}$ of the time-varying constrained control case.
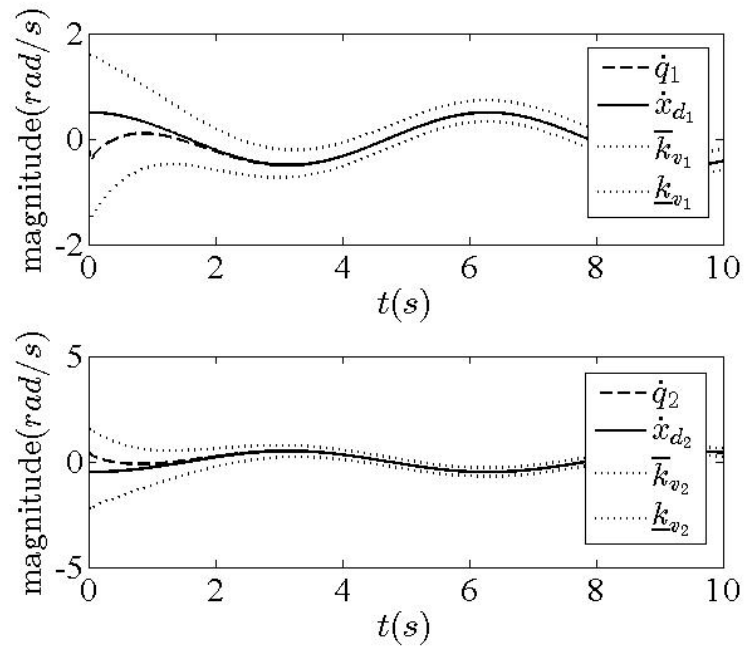

Fig. 8. Desired trajectory $\dot{\boldsymbol{x}}_{\boldsymbol{d}}$ and actual trajectory $\dot{\boldsymbol{q}}$ of joint velocities, with the velocity bounds $\overline{\boldsymbol{k}}_{\boldsymbol{v}}$, and $\underline{\boldsymbol{k}}_{\boldsymbol{v}}$ for the timevarying constrained control case. 

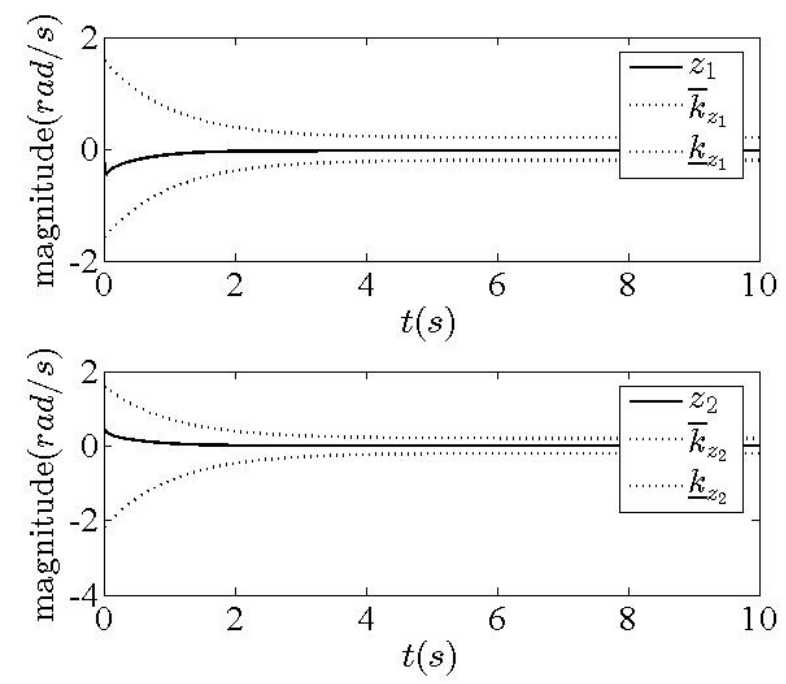

Fig. 9. Trajectory of error function $\boldsymbol{z}$, with constraints $\overline{\boldsymbol{k}}_{z}$, and $\underline{\boldsymbol{k}}_{z}$ for the time-varying constrained control case.

The tracking performance of the robotic system under time-varying constrained controller is illustrated in Figs 7 - 9. It is observed from the results that all signals track the desired trajectories successfully. Figure 7 shows position tracking trajectories. The trajectories for velocity tracking are illustrated in Figs. 8, 9. As it is obvious in these figures, error signals never violate the constraints even if errors are growing close to their bounds. The sBLF as discussed in the Introduction and the control design is responsible for such control effects. It can be observed from Figs. 7-9 that under the proposed controller, good tracking performance is achieved while the error signals never transgress the constraint sets.

\subsection{Third case study}

In this case study, we compare the presented sBLF in this paper with the available logarithm BLF. To this end, we employed the same logarithm BLF, $\boldsymbol{V}_{L}$, as presented in [9] with $\left|\boldsymbol{z}_{\boldsymbol{i}}\right| \leq \boldsymbol{k}_{\boldsymbol{c} i}$ for $\boldsymbol{i}=1,2, \ldots, \boldsymbol{n}$. The control is chosen as,

$$
\tau=-\hat{\boldsymbol{\omega}}^{T} \boldsymbol{h}-\hat{\boldsymbol{\Gamma}} \tanh \left(\frac{\boldsymbol{z}}{\boldsymbol{\varphi}}\right)-\boldsymbol{c}_{2} z-\sum_{i=1}^{n} \frac{\boldsymbol{k}_{1 i} \boldsymbol{e}_{i}}{\sqrt{1+\boldsymbol{e}_{i}^{2}}}-\sum_{i=1}^{n} \frac{1}{\boldsymbol{k}_{c i}{ }^{2}-\boldsymbol{z}_{i}^{2}}\left(\boldsymbol{c}_{3 i} z_{i}+\boldsymbol{a}_{i}-\dot{\alpha}_{i}\right),
$$

and the adaptive laws are chosen the same with (20), and (21). Then, considering $\ln \left(\boldsymbol{k}_{c i}{ }^{2} /\left(\boldsymbol{k}_{c i}{ }^{2}-\boldsymbol{z}_{i}{ }^{2}\right)\right) \leq \boldsymbol{z}_{i}{ }^{2} /\left(\boldsymbol{k}_{c i}{ }^{2}-\boldsymbol{z}_{i}{ }^{2}\right)$ and applying the same calculation as in Section 3, it can show that $\dot{V}_{L} \leq-\bar{\alpha} V_{L}+\bar{\beta}$ with the same $\bar{\alpha}$ and $\bar{\beta}$ as in (24), and (25), respectively.

Now, we are ready to obtain the velocity error $z$ for the logarithm based BLF and the presented SBLF. Similar to the proof of Theorem 1 , by denoting $\boldsymbol{\zeta}=\overline{\boldsymbol{\beta}} / \overline{\boldsymbol{\alpha}}$, it can satisfy that $\boldsymbol{V}_{\boldsymbol{L}}(\boldsymbol{t}) \leq \boldsymbol{\zeta}+\boldsymbol{V}_{\boldsymbol{L}}(0) \exp (-\overline{\boldsymbol{\alpha}} \boldsymbol{t}) \leq \boldsymbol{\zeta}+\boldsymbol{V}_{\boldsymbol{L}}(0)$. Thus, it can be obtained that $1 / 2 \sum_{i=1}^{n} \ln \left(\boldsymbol{k}_{c i}{ }^{2} / \boldsymbol{k}_{c i}{ }^{2}-\boldsymbol{z}_{i}{ }^{2}\right) \leq \boldsymbol{V}_{\boldsymbol{L}} \leq \boldsymbol{\zeta}+\boldsymbol{V}_{\boldsymbol{L}}(0)$. Then, by doing some manipulation, the velocity error signal in the logarithm based BLF can be stated as,

$$
z_{i} \leq \boldsymbol{k}_{c i} \sqrt{1-\exp \left(-2\left(\zeta+V_{L}(0)\right)\right)}
$$

To obtain the velocity error of the sBLF, considering $\boldsymbol{V}(\boldsymbol{t}) \leq \boldsymbol{\zeta}+\boldsymbol{V}(0) \exp (-\overline{\boldsymbol{\alpha}} \boldsymbol{t}) \leq \boldsymbol{\zeta}+\boldsymbol{V}(0)$, it is easy to obtain that $\sec \left(\Lambda_{i}\right)-1 \leq \boldsymbol{V} \leq \boldsymbol{\zeta}+\boldsymbol{V}(0)$. This can lead to the velocity error signal in the sBLF having the response as, 


$$
\boldsymbol{z}_{i} \leq \boldsymbol{k}_{c i} \sqrt{\frac{2}{\pi} \sec ^{-1}(\zeta+\boldsymbol{V}(0)+1)}
$$

Now, we are ready to perform a numerical simulation for this case study. A two-link robotic system with the same parameters with the previous case study is considered for simulation. The constrained error was chosen as $\boldsymbol{k}_{c i}=2.5$, and the control parameters $c_{1 i}$ were selected as $c_{1 i}=2$, for $\boldsymbol{i}=1,2$. Other simulation parameters were the same as for the first simulation. The simulation figures are illustrated in Figs. $10-12$.
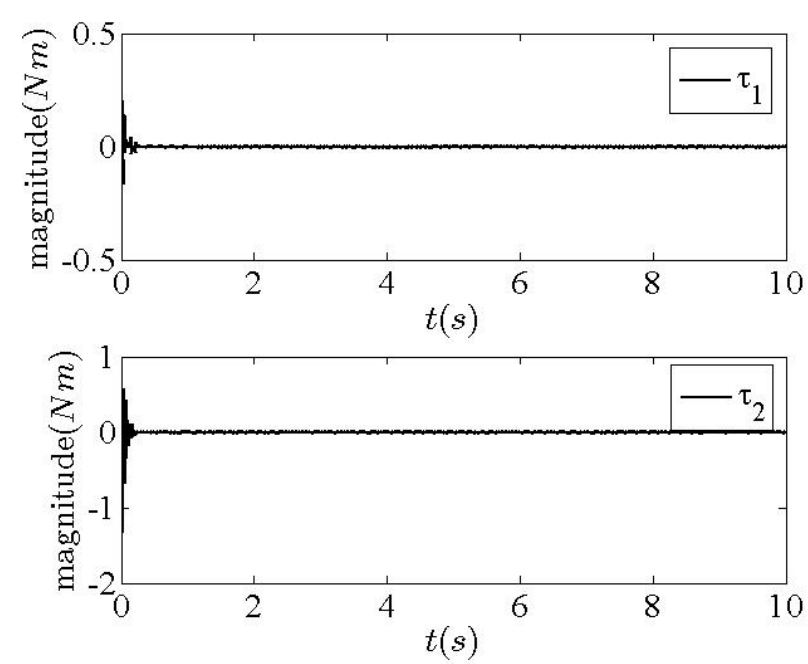

Fig. 10. Trajectory of corresponding sBLF control input term.
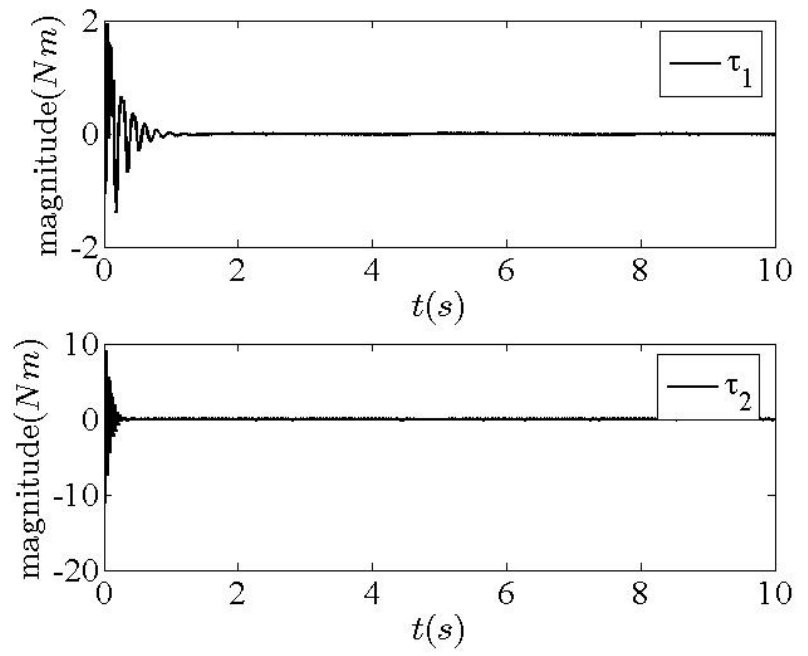

Fig. 11. Trajectory of corresponding logarithm BLF control input term.
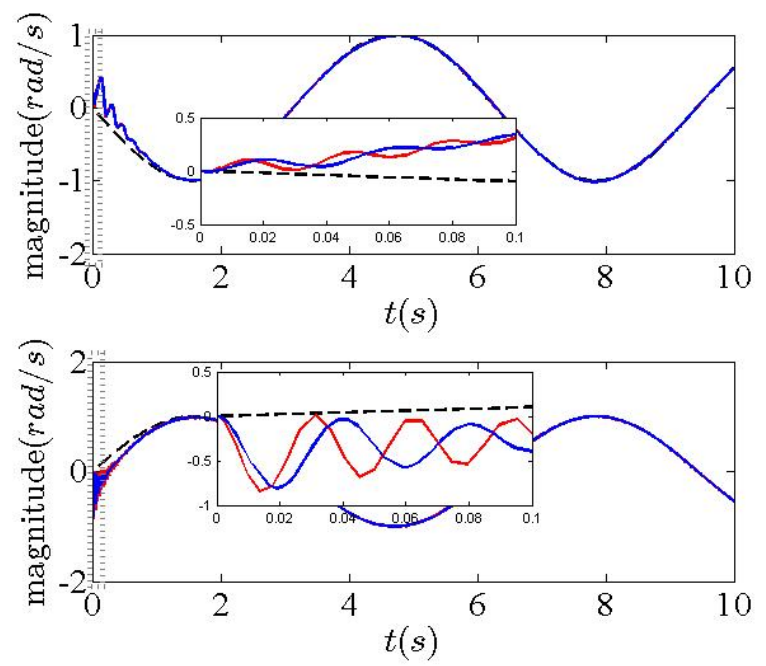

Fig. 12. Trajectory of joint velocity for both BLF types: the desired signal (black) versus the SBLF signal (red), and the logarithm BLF signal (blue).

Figs. 10, and 11 show the trajectories of the corresponding sBLF, and logarithm BLF term in the control input. These terms are the last terms in the control laws in (19) and (34), i.e., $\sum_{i=1}^{n} \pi\left(\boldsymbol{c}_{3 i} z_{i}+\boldsymbol{a}_{i}-\dot{\alpha}_{i}\right) \Upsilon_{i} / \boldsymbol{k}_{c i}{ }^{2}$ for the sBLF control, and $\sum_{i=1}^{n}\left(c_{3 i} z_{i}+a_{i}-\dot{\alpha}_{i}\right) / \boldsymbol{k}_{c i}{ }^{2}-z_{i}{ }^{2}$ for the logarithm BLF control. As shown in these figures using the presented sBLF, a smaller amount of control is required to ensure the constrained velocity control. By that means, using the presented method, less energy is used to perform the same task. Figure 12 shows the tracking of velocities using both methods. This figure illustrates that 
efficient tracking of the desired trajectory can be achieved by both methods.

\section{Conclusion}

This paper presents an adaptive neural control methodology under the existence of velocity constraints and input saturation for robotic systems. A novel secant type barrier Lyapunov function, named sBLF, was introduced to ensure that the velocity constraints were not violated. Input saturation characteristics were properly compensated, and radial basis function neural networks were adopted to cope with the system uncertainties. Using the presented approach, the tracking errors converge to a small neighborhood around zero, and all the signals of the closed-loop system are semi-globally uniformly ultimately bounded. Under the proposed control, extra steps on error transformation or transforming the original constrained system into an equivalent unconstrained one are removed and hence the constraints are directly exploited in the control design. The performance of the proposed control has been established with theoretical analysis and has also been verified by simulation study on a 2-DOF robotic manipulator system. The object of future work is to implement the impedance controller into the proposed control to design a safe and constrained environment- robot interaction control. In addition, in practice the proposed method can be used in robotic systems where constraints on velocity variables are required. Robot dynamics and interaction forces, however, are not known. Furthermore, the ability to provide safety enables the method to be suitable for recent social applications besides the conventional industrial ones. Robotic surgery and the safe robotic rehabilitation are examples of recent practical applications.

\section{References}

[1] K.B. Ngo, R. Mahony, Z.-P. Jiang, Integrator backstepping design for motion systems with velocity constraint, 5th Asian Control ConferenceMelbourne, Victoria, Australia, 2004.

[2] K.B. Ngo, R. Mahony, Z.-P. Jiang, Integrator backstepping using barrier functions for systems with multiple state constraints, 44th IEEE Conference on Decision and Control, 2005 and 2005 European Control Conference. CDC-ECC'05, IEEE, 2005, pp. 8306-8312.

[3] K.P. Tee, S.S. Ge, F.E.H. Tay, Adaptive control of electrostatic microactuators with bidirectional drive, IEEE Transactions on Control Systems Technology, 17 (2009) 340-352.

[4] K.P. Tee, S.S. Ge, E.H. Tay, Barrier Lyapunov functions for the control of output-constrained nonlinear systems, Automatica, 45 (2009) $918-927$.

[5] D. Panagou, D.M. Stipanović, P.G. Voulgaris, Distributed coordination control for multi-robot networks using Lyapunov-like barrier functions, IEEE Transactions on Automatic Control, 61 (2016) 617-632.

[6] Y.-J. Liu, S. Tong, Barrier Lyapunov functions for Nussbaum gain adaptive control of full state constrained nonlinear systems, Automatica, 76 (2017) 143152.

[7] Y.-J. Liu, S. Tong, Barrier Lyapunov functions-based adaptive control for a class of nonlinear pure-feedback systems with full state constraints, Automatica, 64 (2016) 70-75.

[8] Z.-L. Tang, S.S. Ge, K.P. Tee, W. He, Robust adaptive neural tracking control for a class of perturbed uncertain nonlinear systems with state constraints, IEEE Transactions on Systems, Man, and Cybernetics: Systems 46 (2016) 1618 - 1629.

[9] W. He, Y. Chen, Z. Yin, Adaptive neural network control of an uncertain robot with full-state constraints, IEEE Transactions on Cybernetics, 46 (2016) 620629.

[10] Z.-L. Tang, S.S. Ge, K.P. Tee, W. He, Adaptive neural control for an uncertain robotic manipulator with joint space constraints, International Journal of Control, 89 (2016) 1428-1446.

[11] W. He, Y. Dong, Adaptive fuzzy neural network control for a constrained robot using impedance learning, IEEE Transactions on Neural Networks and Learning Systems, inpress; DOI: 10.1109/TNNLS.2017.2665581 (2017).

[12] W. He, S.S. Ge, Cooperative control of a nonuniform gantry crane with constrained tension, Automatica, 66 (2016) 146-154.

[13] X. Jin, Fault tolerant finite-time leader-follower formation control for autonomous surface vessels with LOS range and angle constraints, Automatica, 68 (2016) 228-236.

[14] H. Habibi, H.N. Rahimi, I. Howard, Constrained Control of Wind Turbines for Power Regulation in Full Load Operation, Proceedings of Asian Control Conference Gold Coast, Australia, 2017.

[15] Y. Yang, C. Hua, X. Guan, Adaptive fuzzy synchronization control for networked teleoperation system with input and multi-state constraints, Journal of the Franklin Institute, 353 (2016) 2814-2834.

[16] C. Wen, J. Zhou, Z. Liu, H. Su, Robust adaptive control of uncertain nonlinear systems in the presence of input saturation and external disturbance, IEEE Transactions on Automatic Control, 56 (2011) 1672-1678.

[17] W. He, Y. Dong, C. Sun, Adaptive neural impedance control of a robotic manipulator with input saturation, IEEE Transactions on Systems, Man, and Cybernetics: Systems, 46 (2016) 334-344.

[18] Z. Chen, Z. Li, C.P. Chen, Adaptive neural control of uncertain MIMO nonlinear systems with state and input constraints, IEEE transactions on neural networks and learning systems, 28 (2017) 1318-1330.

[19] T. Guo, X. Wu, Backstepping control for output-constrained nonlinear systems based on nonlinear mapping, Neural Computing and Applications, 25 (2014) $1665-1674$.

[20] S. Tong, S. Sui, Y. Li, Fuzzy adaptive output feedback control of MIMO nonlinear systems with partial tracking errors constrained, IEEE Transactions on Fuzzy Systems, 23 (2015) 729-742.

[21] W. Meng, Q. Yang, J. Si, Y. Sun, Adaptive Neural Control of a Class of Output-Constrained Nonaffine Systems, IEEE Transactions on Cybernetics, 46 (2016) 85-95.

[22] M.M. Polycarpou, P.A. Ioannou, A robust adaptive nonlinear control design, American Control Conference, IEEE, 1993, pp. 1365-1369.

[23] S.S. Ge, C.C. Hang, T.H. Lee, T. Zhang, Stable adaptive neural network control, Springer Science \& Business Media2013.

[24] J. Liu, Radial Basis Function (RBF) neural network control for mechanical systems: design, analysis and Matlab simulation, Springer Science \& Business Media2013.

[25] A. Kurdila, F.J. Narcowich, J.D. Ward, Persistency of excitation in identification using radial basis function approximants, SIAM Journal on Control and Optimization, 33 (1995) 625-642.

[26] R.M. Sanner, J.-J.E. Slotine, Gaussian networks for direct adaptive control, IEEE Transactions on Neural Networks, 3 (1992) 837-863. 
[27] Y. Song, L. Liang, M. Tan, Neuroadaptive Power Tracking Control of Wind Farms Under Uncertain Power Demands, IEEE Transactions on Industrial Electronics, 64 (2017) 7071-7078.

[28] S. Gao, B. Ning, H. Dong, Fuzzy dynamic surface control for uncertain nonlinear systems under input saturation via truncated adaptation approach, Fuzzy Sets and Systems, 290 (2016) 100-117.

[29] W. He, X. He, S.S. Ge, Vibration control of flexible marine riser systems with input saturation, IEEE/ASME Transactions on Mechatronics, 21 (2016) 254265.

[30] Y. Li, S. Tong, T. Li, Composite adaptive fuzzy output feedback control design for uncertain nonlinear strict-feedback systems with input saturation, IEEE Transactions on Cybernetics, 45 (2015) 2299-2308.

[31] K.P. Tee, B. Ren, S.S. Ge, Control of nonlinear systems with time-varying output constraints, Automatica, 47 (2011) 2511-2516.

[32] G.-X. Wen, C.P. Chen, Y.-J. Liu, Z. Liu, Neural-network-based adaptive leader-following consensus control for second-order non-linear multi-agent systems, IET Control Theory \& Applications, 9 (2015) 1927-1934.

[33] H.N. Rahimi, I. Howard, L. Cui, Neural Adaptive Assist-As-Needed Control for Rehabilitation Robots, Australasian Conference on Robotics and Automation (ACRA)Brisbane, Australia, 2016.

[34] H. Rahimi, M. Nazemizadeh, Dynamic analysis and intelligent control techniques for flexible manipulators: a review, Advanced Robotics, 28 (2013) $63-76$.

[35] W. He, Z. Yan, C. Sun, Y. Chen, Adaptive Neural Network Control of a Flapping Wing Micro Aerial Vehicle With Disturbance Observer, IEEE Transactions on Cybernetics, 47 (2017) 3452-3465.

[36] Y.J. Liu, S.M. Lu, D.J. Li, S.C. Tong, Adaptive controller design-based ABLF for a class of nonlinear time-varying state constraint systems, IEEE Transactions on Systems, Man, and Cybernetics: Systems, 47 (2017) 1546-1563. 